\title{
Effects of Initial Radius on the Propagation of Premixed Flame Kernels in a Turbulent Environment
}

\author{
${ }^{1}$ M. Klein ${ }^{*},{ }^{2}$ N. Chakraborty, ${ }^{3}$ K.W. Jenkins, and ${ }^{2}$ R. S. Cant \\ ${ }^{1}$ Technische Universitaet Darmstadt \\ Institut fuer Energie - und Kraftwerkstechnik \\ Petersenstr. 30 \\ D-64287 Darmstadt \\ Tel +49(0) 6151164103 \\ Fax +49(0) 6151166555 \\ Emailkleinm@hrzpub.tu-darmstadt.de \\ ${ }^{2}$ Cambridge University Engineering Department \\ Trumpington Street, Cambridge, CB2 1PZ,UK \\ ${ }^{3}$ School of Engineering, Cranfield University, \\ Bedford, MK43 0AL, UK
}

\footnotetext{
* Corresponding author
} 


\begin{abstract}
The effects of mean curvature on the propagation of turbulent premixed flames have been investigated using three-dimensional Direct Numerical Simulations (DNS) with single step Arrhenius type chemistry in the thin reaction zones regime. A number of spherical flame kernels with different initial radius have been studied under identical conditions of turbulence and thermo-chemistry. A statistically planar turbulent back-to-back flame has been simulated as a special case of a spherical kernel in the limit of infinite kernel radius. Statistical analysis in terms of standard and joint probability density functions (pdfs) clearly indicates that the mean curvature of the flame kernel configuration has a major influence on the propagation behaviour of the flame. For the planar flame configuration the density-weighted displacement speed is found to be fairly constant throughout the flame brush, in good agreement with previous DNS results. By contrast, for the flame kernel configuration the density-weighted displacement speed is found to vary strongly through the flame brush, changing from values on the order of the corresponding laminar flame speed near the fresh gas side to considerably smaller values near the burned gas side. The joint pdfs of displacement speed and its components with curvature are extensively studied, allowing for an explanation of the observed phenomena in terms of local flame geometry and its interaction with the turbulent flow field.
\end{abstract}




\section{INTRODUCTION}

Turbulent premixed combustion has wide applications in a variety of engineering devices including gas turbines and spark ignition engines. Since the majority of combustion systems operate in a turbulent flow environment, it is necessary to consider the turbulence and its interaction with the flame. Using Direct Numerical Simulations (DNS), it is possible to simulate a turbulent flame without the need for modelling of the flow field. Thereby useful information can be extracted to support combustion modelling based on Reynolds Averaged Navier Stokes equations (RANS) and Large Eddy Simulation (LES) ${ }^{1}$. Ideally, combustion DNS should address the three-dimensional nature of the turbulence together with detailed chemistry, but this remains extremely expensive even with present day computational resources. As a consequence, most combustion DNS has been carried out either in two dimensions with detailed chemistry ${ }^{2-4}$ or in three dimensions with simplified chemistry. ${ }^{5-9}$ As an alternative, reduced chemical mechanisms have recently been used in simulations of flame development in $3 \mathrm{D},{ }^{10-13}$ and comparisons of $2 \mathrm{D}$ versus $3 \mathrm{D}$ simulation results have recently been made for spherical flame kernels. $^{10,13}$ Since the flame-turbulence interaction is the principal interest of this work, and since 2D turbulence is physically different from 3D turbulence, the focus of this work is on 3D turbulence with simplified chemistry.

Displacement speed is a central quantity for the understanding and modelling of turbulent premixed flames, and it appears in the governing equations for common model formulations such as the Flame Surface Density (FSD) approach ${ }^{14}$ and the $G$-equation approach. ${ }^{15}$ In many practical combustion problems the smallest scales of turbulence can 
enter into the flame structure and cause unsteady fluctuations in the preheat zone ahead of the thin reaction layer. Peters ${ }^{15}$ named this regime of premixed turbulent combustion the 'thin reaction zones regime'. In this particular parameter range, $S_{d}$ is strongly dependent on the local flame curvature.

Many previous DNS studies have been concerned with statistically planar flames which by definition have zero mean curvature. ${ }^{2-7}$ By contrast, the flame kernel is an example of a turbulent flame that has significant mean curvature. Previous work on flame kernels has focussed on ignition ${ }^{16}$ and the evaluation of flame surface properties such as FSD, flame curvature and the flame normal vector. ${ }^{8,9,11,12}$ A qualitative comparison between flame kernel DNS data and experimental results has also been made in recent studies. ${ }^{17,18}$

For statistically planar flames, the behaviour of the displacement speed in response to flame curvature has been extensively studied ${ }^{2-4}$. By contrast, displacement speed behaviour in the presence of intrinsic mean flame curvature has rarely been addressed. Recently, van Oijen et al. ${ }^{13}$ examined stretch effects on the local burning rate and assessed different possible flamelet models with respect to DNS data. It was pointed out that burning rate models involving Markstein numbers and designed for small stretch rates may not produce the correct propagation behaviour in the presence of large curvature stretch, such as that induced by spherical flame kernels.

As far as the authors are aware, the effect of initial flame kernel radius on the subsequent flame propagation has not been studied to date using DNS. To investigate this effect in 
detail, several DNS of flame kernels with different initial radius have been carried out. At the same time, as a limiting case, a statistically planar turbulent back-to-back flame has been simulated. Significant differences have been found in the statistical behaviour of the displacement speed. The purpose of this study is twofold:

1. To study the difference in both local and mean displacement speed behaviour in response to mean flame curvature.

2. To identify the physical mechanisms responsible for the observed behaviour

3. To identify the implications for flamelet based turbulent combustion modelling, especially in the thin reaction zones regime. ${ }^{15}$

The rest of the paper is organised as follows. The necessary mathematical background and numerical implementation are presented in Section II. This is followed in Section III by the presentation and discussion of the results. The main findings are summarised in the fourth and final section of the paper.

\section{MATHEMATICAL BACKGROUNDAND NUMERICAL IMPLEMENTATION} Assuming single-step Arrhenius reaction kinetics it is possible to account for the chemical state of the system in terms of a reaction progress variable $c$ defined as

$$
c=\frac{Y_{P}-Y_{P 0}}{Y_{P \infty}-Y_{P 0}}
$$

where $Y_{P}$ is the product mass fraction and subscripts 0 and $\infty$ are used to denote unburned reactants and completely burned products respectively. The transport equation for the reaction progress variable is:

$$
\rho \frac{\partial c}{\partial t}+\rho u_{j} \frac{\partial c}{\partial x_{j}}=\frac{\partial}{\partial x_{j}}\left(\rho D \frac{\partial c}{\partial x_{j}}\right)+\dot{w}
$$


where $\rho$ is the density, $u_{j}$ is the fluid velocity component in the $j^{\text {th }}$ direction, $D$ is the species diffusivity and $\dot{w}$ is the reaction rate. In kinematic form ${ }^{2}$ eq. 2 may be written for a given progress variable isosurface $c=c^{*}$ as:

$$
\left.\frac{\partial c}{\partial t}\right|_{c=c^{*}}+\left.u_{j} \frac{\partial c}{\partial x_{j}}\right|_{c=c^{*}}=S_{d} \mid \nabla c \|_{c=c^{*}}
$$

where $S_{d}$ is the displacement speed of the $c=c^{*}$ isosurface. Comparing eq. 2 and eq. 3 the displacement speed is given by:

$$
S_{d}=\left.\frac{\dot{w}+\nabla \cdot(\rho D \nabla c)}{\rho|\nabla c|}\right|_{c=c^{*}}
$$

As can be seen from eq.4, the displacement speed is a result of the interaction between reaction and diffusion within the flame, and $S_{d}$ is the speed at which the flame moves relative to the flow in the direction of the local flame normal vector. The $i^{\text {th }}$ component of the local flame normal is given as:

$$
N_{i}=-\frac{1}{|\nabla c|} \frac{\partial c}{\partial x_{i}}
$$

The progress variable gradient magnitude $|\nabla c|$ which appears eqs.3, 4 and 5 is an important quantity and will be referred to as the Surface Density Function (SDF) following Kollmann and Chen. ${ }^{19}$ The mean curvature on a $c=c^{*}$ isosurface can be expressed as:

$$
\kappa_{m}=\left.\frac{1}{2} \frac{\partial N_{i}}{\partial x_{i}}\right|_{c=c^{*}}
$$

In the present convention, the local flame normal vector points towards the reactants and positive mean curvature is defined as convex towards the reactants. 
Local turbulence plays an important role in determining $S_{d}$. Under the influence of turbulence, the flame becomes stretched and curved and the resulting local geometry effects come into $S_{d}$ through the diffusion term $\nabla \cdot(\rho D \nabla c)$ and the $\operatorname{SDF}|\nabla c|$. The effects of mean curvature on the displacement speed represent the main area of interest in the present study. It is helpful to split the diffusion term into normal and tangential components $^{3,7}$ :

$$
\nabla \cdot(\rho D \nabla c)=\vec{N} \cdot \nabla(\rho D \vec{N} \cdot \nabla c)-\rho D \nabla \cdot \vec{N}|\nabla c|
$$

Similarly the displacement speed $S_{d}$ can be decomposed into a reaction component $S_{r}$, a normal diffusion component $S_{n}$ and a tangential diffusion component $S_{t}$

$$
S_{d}=S_{r}+S_{n}+S_{t}
$$

where $S_{r}, S_{n}$ and $S_{t}$ are given by:

$$
S_{r}=\left.\frac{\dot{w}}{\rho|\nabla c|}\right|_{c=c^{*}} ; S_{n}=\left.\frac{\vec{N} \cdot \nabla(\rho D \vec{N} \cdot \nabla c)}{\rho|\nabla c|}\right|_{c=c^{*}} ; S_{t}=-2 D \kappa_{m}
$$

It is clear from eq. 8ii that the tangential diffusion component $S_{t}$ is directly proportional to the negative of mean curvature. It is worth noting that the SDF $|\nabla c|$ occurs in the denominator of the expressions for both the normal diffusion component $S_{n}$ and the reaction component $S_{r}$, resulting in significant potential for the SDF to influence the displacement speed statistics. $^{7}$

DNS has been carried out using a three-dimensional finite-difference code called SENGA $^{20}$ which solves the compressible Navier-Stokes equations augmented by a transport equation for the reaction progress variable. All first and second order 
derivatives are evaluated using tenth order explicit central differencing in the interior of the domain, with gradual reduction to $2^{\text {nd }}$ order one-sided differencing as the outlet boundaries are approached. Time stepping is carried out using a low storage $3^{\text {rd }}$ order Runge Kutta scheme ${ }^{21}$. A pseudo spectral method ${ }^{22}$ is used to initialise the velocity field by generating a good approximation to homogeneous isotropic incompressible turbulence. Boundary conditions are specified using the Navier Stokes Characteristic Boundary Condition (NSCBC) formulation as suggested by Poinsot and Lele. ${ }^{23}$ For the flame kernel configuration, either a standard non-reflecting outflow or a partially reflecting inflow condition is used for all faces of the cubic domain depending on the local sign of the velocity normal to the boundary. For the planar flame configuration, the same boundary condition is applied to two opposite faces of the cubic domain, while periodic boundary conditions are applied to the transverse faces.

In all cases the premixed flame is initialised using a pre-computed laminar flame solution with identical thermochemical parameters. In order to ensure adequate resolution, about 10 grid points are used to resolve the laminar thermal flame thickness $\delta_{t h}$ given by:

$$
\delta_{t h}=\frac{\left(T_{a d}-T_{0}\right)}{\operatorname{Max}\left|\frac{\partial \hat{T}}{\partial n}\right|}
$$

where $n$ is the normal direction of flame propagation, $\hat{T}$ is the dimensional temperature, and $T_{0}$ and $T_{a d}$ are the fresh gas and adiabatic flame temperatures respectively. For the sake of simplicity, the specific heats $C_{P}$, viscosity $\mu$, thermal conductivity $\lambda$ and density weighted mass diffusivity $\rho D$ are assumed to be constant and independent of 
temperature. The Lewis number is unity for all cases. The turbulence Reynolds number is defined as $\operatorname{Re}_{t}=\rho_{0} u^{\prime} l_{t} / \mu$, and the nominal Reynolds number used to nondimensionalise the momentum equations is defined as $\operatorname{Re}_{S_{L}}=\rho_{0} S_{L} L / \mu$, where $\rho_{0}$ is the fresh gas density, $u^{\prime}$ is the turbulent velocity fluctuation magnitude, $l_{t}$ is the integral length scale of the turbulence, $S_{L}$ is the unstrained laminar flame speed and $L$ is a reference length scale with $L \approx 10 \delta_{t h}$, where $\delta_{t h}$ is the laminar thermal flame thickness. The last two $\left(S_{L}\right.$ and $\left.L\right)$ are taken as the reference velocity and length scales respectively and are used to normalise other relevant quantities. The mesh size for all cases is $230 \times 230 \times 230$ giving $\operatorname{Re}_{t}=32$ and $\operatorname{Re}_{S_{L}}=25$, and the computational domain is chosen to be a cube of side $2.4 \mathrm{~L}$ which allows ample space for the flame kernel to grow. A list of all numerical and physical parameters is given in Table I.

\section{RESULTS \& DISCUSSION}

For the values of turbulent Reynolds number $\left(\operatorname{Re}_{t}\right)$, length scale ratio $\left(L_{11} / \delta_{L}\right)$ and velocity scale ratio $\left(u^{\prime} / S_{L}\right)$ used in the present study (see Table I), the turbulent combustion in all cases occurs within the thin reaction zones regime. ${ }^{15}$ In Table I, Case A indicates a pair of planar back-to-back flames which corresponds to a flame kernel with infinite radius. For the purpose of this study, results corresponding to cases $\mathrm{A}, \mathrm{C}$ and F will mainly be presented. Similar qualitative trends are observed for the other cases. Statistical information has been obtained at a simulation time of three initial eddy turnover times $\left(\tau_{f 0}=L_{11} / u^{\prime}\right)$ which corresponds to about 1.2 chemical times $\tau_{c}$. 
Here $\tau_{c}=\delta_{Z} / S_{L}$, where $\delta_{Z}=D^{+} / s_{d}$ and where $D^{+}$and $s_{d}$ are the diffusivity and burning velocity evaluated at the reaction zone. ${ }^{15}$

Contours of reaction progress variable in the $x-z$ plane at the location $y=0.5$ are presented in Figs. 1a-c for the cases A, C and F respectively. Note that in case A (Fig. 1a) the boundaries are periodic in the $y$ and $z$ directions. It is evident in all three cases that the preheat zone $(c<0.5)$ is highly deformed and the contours are not parallel. However, in the reaction zone $(0.7<c<0.9)$ the contours do remain parallel. This is typical of the thin reaction zones regime where turbulent eddies enter into the preheat zone and causes significant fluctuations. ${ }^{15}$ Fig. 1 shows furthermore that the progress variable contours are increasingly positively curved for the flames with smaller radius.

In order to understand the flame propagation it is helpful to look at the reaction-diffusion balance across the flame brush (see eq. 4). The mean behaviour of the reaction rate $\dot{w}$ and the molecular diffusion rate $(\nabla \cdot(\rho D \nabla c))$ across the flame brush for cases $\mathrm{A}, \mathrm{C}$ and $\mathrm{F}$ is presented in Fig. 2a-c respectively. For the purpose of subsequent analysis, the components of the local molecular diffusion rate in the flame normal $\operatorname{direction}(\vec{N} \cdot \nabla(\rho D \vec{N} \cdot \nabla c))$ and in the flame tangential direction $(-\rho D(\nabla \cdot \vec{N})|\nabla c|)$ are also shown in Fig. 2. It is important to note that these quantities are plotted as a conditional average on a given $c$ isosurface, in the manner previously used by Boger et al. ${ }^{24}$ and Chakraborty and Cant. ${ }^{7}$ The profile of mean reaction rate in all three cases increases sharply towards the burned gas side of the flame and attains a maximum value at roughly $c=0.8$. The mean values of both $\nabla \cdot(\rho D \nabla c)$ and $\vec{N} . \nabla(\rho D \vec{N} . \nabla c)$ are positive 
towards the fresh gas side with a local maximum some way ahead of the middle of the flame, and both are negative towards the burned gas side with a local minimum close to the maximum reaction rate location. The quantitative behaviour of the molecular diffusion rate and its components is different for different flame radii although the qualitative behaviour remains the same. The planar flame (case A, Fig. 2a) corresponds to infinite flame radius (i.e. zero mean curvature) which leads to a negligible contribution from the tangential component $(-\rho D(\nabla \cdot \vec{N})|\nabla c|)$, and hence the mean values of $\nabla \cdot(\rho D \nabla c)$ and $\vec{N} . \nabla(\rho D \vec{N} . \nabla c)$ remain very close to each other throughout the flame (see eq.7). With decreasing flame radius the mean curvature of the flame kernel becomes increasingly positive and therefore the mean contribution of $(-\rho D(\nabla \cdot \vec{N})|\nabla c|)$ becomes increasingly negative throughout the flame, as is evident from Figs. $2 b$ and c. As a result of this, the difference between the mean values of $\nabla \cdot(\rho D \nabla c)$ and $\vec{N} . \nabla(\rho D \vec{N} . \nabla c)$ increases with decreasing kernel radius.

The density weighted displacement speed quantities $\rho S_{d}$ and $\rho\left(S_{r}+S_{n}\right)$ are central to the understanding and flamelet based modelling of turbulent premixed combustion. In the context of RANS, surface averaged values of $\rho S_{d}$ and $\rho\left(S_{r}+S_{n}\right)$ on a specified $c=c^{*}$ isosurface are given by: ${ }^{25}$

$$
\begin{gathered}
\left\langle\rho S_{d}\right\rangle_{S}=\frac{\left\langle\dot{w}+\nabla \cdot(\rho D \nabla c) \mid c=c^{*}\right\rangle}{\left\langle\mid \nabla c \| c=c^{*}\right\rangle} \\
\left\langle\rho\left(S_{r}+S_{n}\right)\right\rangle_{S}=\frac{\left\langle\dot{w}+\vec{N} \cdot \nabla(\rho D \vec{N} \cdot \nabla c) \mid c=c^{*}\right\rangle}{\left\langle\mid \nabla c \| c=c^{*}\right\rangle}
\end{gathered}
$$


where angled brackets indicate an ensemble averaging operation. Often $\left\langle\rho S_{d}\right\rangle_{S}$ is approximated as $\rho_{0} S_{L}$, and the validity of this assumption will be discussed in the context of Figs. 3 and 4.

Profiles of the mean reaction-diffusion imbalance $\dot{w}+\nabla \cdot(\rho D \nabla c)$ and the mean reactionnormal diffusion imbalance $\dot{w}+\vec{N} . \nabla(\rho D \vec{N} . \nabla c)$ are presented in Figs. 3a-c for cases A, C and $\mathrm{F}$ respectively along with the corresponding mean profile of the SDF in the form $\rho_{0} S_{L}|\nabla c|$ (see eq. 4). In all cases, the mean profile of $\rho_{0} S_{L}|\nabla c|$ is skewed towards the burned gas side of the flame with a maximum close to $c \approx 0.65$, similar to previous experimental $^{26-28}$ and DNS studies. ${ }^{7,24,25,29}$ It is evident that in the planar case (case A) the mean values of $\dot{w}+\nabla \cdot(\rho D \nabla c)$ and $\dot{w}+\vec{N} \cdot \nabla(\rho D \vec{N} . \nabla c)$ are very close to each other, and that both quantities have a similar behavioural trend and a similar order of magnitude to $\rho_{0} S_{L}|\nabla c|$. From eq.10 and Fig.3a this indicates that for the planar flame $\left\langle\rho S_{d}\right\rangle_{S} \approx\left\langle\rho\left(S_{r}+S_{n}\right)\right\rangle_{S} \approx \rho_{0} S_{L}$ throughout the flame brush, almost irrespective of the choice of isosurface $c^{*}$. For the spherical flame kernels (see Figs. $3 b$ and $3 c$ ) the situation is different. The mean value of $\dot{w}+\vec{N} . \nabla(\rho D \vec{N} . \nabla c)$ for the kernel cases is greater than $\dot{w}+\nabla \cdot(\rho D \nabla c)$ with an increasing difference for smaller kernel radius owing to the higher negative contribution of the tangential component $(-\rho D(\nabla \cdot \vec{N})|\nabla c|)$ (see Fig. 2 and eq.7). Figs. 3b-c show that for the kernel cases the mean values of both $\dot{w}+\vec{N} . \nabla(\rho D \vec{N} . \nabla c)$ and $\dot{w}+\nabla(\rho D \nabla c)$ are smaller than the mean value of $\rho_{0} S_{L}|\nabla c|$ across the flame brush which indicates (eq.10) that $\left\langle\rho\left(S_{r}+S_{n}\right)\right\rangle_{S}$ and $\left\langle\rho S_{d}\right\rangle_{S}$ are each smaller than $\rho_{0} S_{L}$. This shows 
clearly that the presence of initial mean curvature has a significant effect on the behaviour of the surface averaged density weighted displacement speed.

The mean profiles of displacement speed and its components $S_{d} / S_{L},\left(S_{r}+S_{n}\right) / S_{L}$ and $S_{t} / S_{L}$, as well as the density-weighted quantities $\rho S_{d} / \rho_{0} S_{L}$ and $\rho\left(S_{r}+S_{n}\right) / \rho_{0} S_{L}$, are shown in Fig. 4a-c for cases A, C and F respectively. For the planar flame (case A) it is evident that the mean variations of $S_{d} / S_{L}$ and $\left(S_{r}+S_{n}\right) / S_{L}$ are almost identical owing to the negligible mean value of the tangential component $S_{t} / S_{L}=-2 D \kappa_{m} / S_{L}$. However the mean value of $S_{d} / S_{L}$ decreases with decreasing kernel radius due to the increasing negative mean value of $S_{t} / S_{L}$, as evident from Fig. 4 and the previous discussion of Figs 2 and 3. Furthermore, it can be seen from Fig. 4a that for the planar flame the mean variation of $\rho S_{d} / \rho_{0} S_{L}$ is almost identical to that of $\rho\left(S_{r}+S_{n}\right) / \rho_{0} S_{L}$, and the mean values remain close to unity throughout the flame brush. On the other hand for the flame kernels (cases $\mathrm{C}$ and $\mathrm{F}$ ) the mean values of $\rho S_{d} / \rho_{0} S_{L}$ decrease from a peak around unity on the fresh gas side to considerably smaller values on the burned gas side, whereas the mean value of $\rho\left(S_{r}+S_{n}\right) / \rho_{0} S_{L}$ remains closer to unity but settles to a smaller value. The mean value of $\rho S_{d} / \rho_{0} S_{L}$ towards the burned gas side is the lowest for the case with the smallest kernel radius (case F, Fig. 4c).

These findings have major implications for turbulent premixed flame modelling, in which a common assumption is that

$$
\left\langle\rho S_{d}\right\rangle_{S}=\rho_{0} S_{L}
$$


It is evident from the above results that indeed this assumption is valid for statistically planar flames because of the negligible contribution from the tangential diffusion component of displacement speed. However eq. 11 may be rendered invalid in a configuration other than a planar flame where the contribution of $\left\langle\rho S_{t}\right\rangle_{S}$ is not negligible. Also, although the mean value of $\rho\left(S_{r}+S_{n}\right)$ remains of the order of $\rho_{0} S_{L}$ in all cases, the surface averaged value $\left\langle\rho\left(S_{r}+S_{n}\right)\right\rangle_{S}$ decreases from $\rho_{0} S_{L}$ with decreasing kernel radius. This, along with the definition of $\left\langle\rho\left(S_{r}+S_{n}\right)\right\rangle_{S}$ in eq. 10ii, indicates that curvature effects on the SDF and $\rho\left(S_{r}+S_{n}\right)$ are responsible for this behaviour. This issue will be revisited in detail below.

The pdfs of normalised displacement speed $\left(S_{d} / S_{L}\right)$ across the flame brush are presented in Figs. 5. In all cases the most probable value of displacement speed is on the order of $S_{L}$. For the planar case (Fig. 5a) the displacement speed pdf broadens and the peak value decreases towards the burned gas side. The tendency of the pdf to broaden is greatest in the planar flame case and least in the smallest kernel case (Fig. 5c). This indicates that the variance of $S_{d}$ is highest in the planar case and decreases with decreasing kernel radius. It can also be seen that the probability of finding high positive values of $S_{d}$ is highest in the planar flame case and lowest in the smallest kernel case. The displacement speed pdfs are consistent with previous planar flame DNS studies $3,7,30$ and the experimental flame kernel investigations of Renou et al. ${ }^{31}$ Comparing Figs. 5a-c it can be seen that the probability of finding a negative value of displacement speed increases with decreasing flame kernel radius. Moreover, the mean value of $S_{d}$ decreases 
with decreasing kernel radius, consistent with Figs. 4a-c. The increased probability of negative $S_{d}$ with decreasing kernel radius has serious consequences for ignition in a turbulent flow. If the kernel radius is smaller than a critical radius the flame will tend to propagate in the opposite direction to the local flame normal, which will lead to shrinkage of the burned gas kernel and once the reaction zone is lost the flame will quench. On the other hand if the flame radius is larger than the critical radius the mean displacement speed will be positive and the kernel will be able to grow, leading to self-sustained combustion. This observation is consistent with the behaviour of flame kernel propagation near the critical radius as observed by Baum and Poinsot ${ }^{16}$ based on twodimensional DNS with simplified chemistry.

In view of these results, it is instructive to investigate the pdfs of the individual components of displacement speed for the different kernel radius cases. The pdfs of the components of displacement speed $\left(S_{r}, S_{n}\right.$ and $\left.S_{t}\right)$ for the $c=0.8$ isosurface - close to the location of maximum reaction rate - are presented in Fig. 6. The pdfs of the reaction component $S_{r}$ (Fig 6a) show a sharp peak at a high positive value, and the form of the pdfs is similar for all three radii. Figure $6 \mathrm{~b}$ shows the corresponding pdfs of the normal diffusion component $S_{n}$, which is sharply peaked at a high negative value in all three cases, consistent with the mean behaviour of the normal molecular diffusion component $\vec{N} . \nabla(\rho D \vec{N} . \nabla c)$ as presented in Fig. 2. Again the form of the pdf is similar for all three radii. Figure 6c shows the pdfs of $S_{t}$ which are markedly different for the three cases. Here, the probability of finding a negative value of $S_{t}$ is lowest in the planar flame case 
and highest in the smallest kernel case, as expected (see eq.8ii). All of these results are qualitatively consistent with previous DNS studies.,

The joint pdfs of displacement speed and curvature are presented in Figs. 7a-c for cases A, C and F respectively. In all three cases the correlation between $S_{d}$ and curvature is negative which is consistent with previous DNS studies with global Lewis number close to unity. ${ }^{7,29}$ According to linearised theory the curvature response of displacement speed can be expressed in terms of the Markstein diffusivity $\left(D_{M}\right)$ as:

$$
S_{d}=\rho_{0} S_{L} / \rho-D_{M} \kappa_{m}
$$

Two-dimensional DNS with detailed chemistry ${ }^{2-4}$ has suggested that the above expression may also be used in the thin reaction zones regime without significant loss of accuracy for small values of curvature-induced stretch. The Markstein diffusivity $D_{M}$ as defined by eq.12 has been evaluated from the DNS results using linear regression analysis and values are presented in Table II. It is evident that $D_{M}$ is positive in all of the cases considered here and that the magnitude of $D_{M}$ decreases with decreasing kernel radius. In order to explain this behaviour it is useful to examine the curvature response of the individual components of displacement speed.

The tangential diffusion component of displacement speed $S_{t}$ is proportional to the negative of local mean curvature (eq. 8ii), and for unity Lewis number flames the diffusivity $D$ does not vary appreciably on a given $c$ isosurface. Thus $S_{t}$ and curvature are deterministically related with a correlation coefficient of -1 . For adiabatic low Mach 
number flames at unity Lewis number (as in the present case) the reaction rate $\dot{w}$ and density $\rho$ remain almost constant on a given $c$ isosurface due to the almost uniform temperature. Therefore the curvature response of $S_{r}$ is entirely governed by the variation of SDF with respect to curvature. Since the reciprocal of SDF can be taken as a measure of local flame thickness, the curvature response of the normal diffusion component of displacement speed $S_{n}$ is governed by the correlation between SDF and curvature, as long as density weighted mass diffusivity $\rho D$ variation is not significant on the given isosurface.

The joint pdfs of SDF and curvature for cases A, C and F are presented in Fig. 8. In the planar flame case (Fig. 8a) it is evident that there are two branches, one showing a negative correlation for positive values of curvature and the other showing a positive correlation for high negative curvatures. The negatively correlating branch becomes stronger with decreasing kernel radius while the positively correlating branch gradually disappears (see Figs. $8 \mathrm{~b}$ and c) since, for smaller kernels, the probability of finding negative curvature decreases with kernel radius.

In order to explain the above behaviour it is helpful to examine the joint pdfs of tangential strain rate and curvature and of dilatation and curvature. Dilatation and tangential strain rate are related according to the expression

$$
\nabla \cdot \vec{u}=a_{T}+a_{n}
$$

where $a_{n}$ and $a_{T}$ are the normal and tangential strain rates given by: 


$$
a_{n}=N_{i} N_{j} \frac{\partial u_{i}}{\partial x_{j}} ; a_{T}=\left(\delta_{i j}-N_{i} N_{j}\right) \frac{\partial u_{i}}{\partial x_{j}}
$$

It is evident from Fig.9 that for all three cases shown, tangential strain rate and curvature are negatively correlated, which is in agreement with previous $\mathrm{DNS}^{7,29,32}$ and experimental studies. ${ }^{31}$ The corresponding joint pdfs between dilatation and curvature are presented in Fig.10, and in all cases dilatation and curvature are seen to be negatively correlated, since negatively (positively) curved regions give rise to a greater (smaller) value of dilatation compared to a planar flame sheet because of focussing (defocussing) of heat. The dilatation-curvature correlation shows evidence of non-linearity, with different slopes for positive and negative curvatures.

The pdfs of normal strain rate for cases A, C and F are presented in Figs. 11a-c. It is evident that the probability of finding a locally compressive (i.e., negative) normal strain rate overwhelms the probability of finding an extensive normal strain rate for all $c$ isosurfaces, implying that $a_{n}=\nabla \cdot \vec{u}-a_{T}<0$ in the mean. This indicates that the flame is aligning itself with the most compressive normal strain rate, which is consistent with previous incompressible two- and three-dimensional DNS studies. ${ }^{5,33}$ Under the action of compressive normal straining the isoscalar lines move closer together leading to a higher value of SDF, and vice versa. The SDF-curvature joint pdf (Fig. 8) is therefore governed by the relative strength of the dilatation-curvature and tangential strain rate-curvature correlations (eq. 12i). Due to the respective negative correlations, small values of both dilatation and tangential strain rate are associated with positive curvatures. Over most of the flame, the tangential strain rate exceeds the local dilatation, as can be observed from 
the normal strain rate pdfs (Fig.11) where the probability of finding negative values of $a_{n}$ is greater than the probability of finding positive values. This is further substantiated by the correlation coefficients between $|\nabla c|$ and tangential strain rate $a_{T}$ presented in column 2 of Table III. As a result of this, flame thickening takes place in regions of positive curvature which leads to the observed negatively-correlating branch of the joint pdfs of SDF and curvature (Fig. 8), consistent with the findings of Chakraborty and Cant. ${ }^{29}$ In negatively curved regions of the flame, dilatation may overcome the tangential strain rate due to local focussing of heat, leading to an extensive normal strain rate. This in turn gives rise to a secondary thickening effect in regions of highly negative curvature as demonstrated by Chakraborty and $\mathrm{Cant}^{29}$ in the case of statistically planar flames. However the probability of finding highly negative curvature decreases with decreasing flame kernel radius, and so the secondary thickening effect gradually disappears with decreasing kernel radius. The correlation coefficients between $|\nabla c|$ and curvature are presented in column 3 of Table III, and indicate an increase in the strength of the negative correlation with decreasing mean kernel radius.

The joint pdfs of the reaction component of displacement speed $S_{r}$ and curvature for cases A, C and F are presented in Fig. 12. These pdfs reflect the effects of the curvature dependence of SDF (see eq.8ii and Figs. 8a-c). For the planar case the joint pdf shows two branches with the positively correlating branch being slightly stronger. With decreasing kernel radius the negatively correlating branch disappears and the correlation between $S_{r}$ and curvature becomes increasingly positive (see Figs. 12b and c). This effect is due to the disappearance of the positively correlating branch in the SDF- 
curvature correlation. A similar trend is observed for all cases, as evident from the correlation coefficients shown in column 4 of Table III.

The corresponding joint pdfs of the normal diffusion component of displacement speed $S_{n}$ and curvature are presented in Fig. 13. For the planar flame (case A, Fig.13a) the joint pdf shows two branches, consistent with the joint pdf of SDF and curvature for the same case (see Fig. 8a). By contrast, in the case of kernels the negatively correlating branch becomes increasingly weak with the decrease in kernel radius (see Figs. $8 \mathrm{~b}$ and c). As a result, the correlation between $S_{n}$ and curvature becomes increasingly positive with the decrease in mean kernel radius. The above behaviour can be explained in the light of the SDF-curvature correlation in the following manner. A decrease in flame thickness induces a high scalar gradient which acts to increase the magnitude of the normal diffusion rate $\vec{N} \cdot \nabla(\rho D \vec{N} \cdot \nabla c)$. In the reaction zone (close to $c=0.8)$ the normal diffusion rate is predominantly negative, and so it becomes more negative with increasing SDF. Therefore a net negative correlation between SDF and curvature in the kernel cases leads to a net positive correlation between $S_{n}$ and curvature with increasing strength with decreasing kernel radius. The correlation coefficients between $S_{n}$ and curvature are presented in column 5 of Table III. The joint pdfs of $S_{r}$ and $S_{n}$ with curvature are in agreement with previous DNS studies for planar flames. ${ }^{3,7,34}$

For the sake of completeness and for the purposes of modelling the joint pdfs of the combined reaction and normal diffusion components of displacement speed and curvature 
are shown in Fig. 14. As kernel radius decreases, $\left(S_{r}+S_{n}\right)$ becomes increasingly positively correlated with curvature. This is not surprising since both $S_{r}$ and $S_{n}$ become increasingly positively correlated with curvature, A similar trend is observed in column 6 of Table III where the correlation coefficients between $\left(S_{r}+S_{n}\right)$ and curvature are presented for different kernel sizes. For the planar flame $\left(S_{r}+S_{n}\right)$ and curvature are weakly correlated which is consistent with Figs. 12a and 13a.

In the flame kernel cases $\left(S_{r}+S_{n}\right)$ is positively correlated with curvature because of the negative correlation between SDF and curvature, which results in a negative correlation between $\left(S_{r}+S_{n}\right)$ and SDF. For this negative correlation, the definition of covariance between $\left(S_{r}+S_{n}\right)$ and $|\nabla c|$ suggests $\left\langle\rho\left(S_{r}+S_{n}\right)|\nabla c|\right\rangle\left\langle\left\langle\rho\left(S_{r}+S_{n}\right)\right\rangle\langle|\nabla c|\rangle\right.$ in the kernel cases. On the other hand, for statistically planar flames a weak correlation between $\left(S_{r}+S_{n}\right)$ and SDF leads to $\left\langle\rho\left(S_{r}+S_{n}\right)|\nabla c|\right\rangle \approx\left\langle\rho\left(S_{r}+S_{n}\right)\right\rangle\langle|\nabla c|\rangle$. This explains the equality between the mean values of $\dot{w}+\vec{N} . \nabla(\rho D \vec{N} . \nabla c)$ and $\rho_{0} S_{L}|\nabla c|$ in the planar flame case and the difference between these quantities in the kernel cases as observed in Fig. 3.

The net correlation between $\left(S_{r}+S_{n}\right)$ and curvature for the kernel cases is important for the modelling of turbulent flame propagation. The total stretch rate induced by flame curvature is given by:

$$
S_{d} \nabla \cdot \vec{N}=\left(S_{r}+S_{n}\right) \nabla \cdot \vec{N}-4 D \kappa_{m}^{2}
$$


where the first term on the right hand side represents the stretch associated with quasilaminar propagation and the last term represents the stretch originating purely due to the geometrical curvature of the flame. As a result of the correlation between $\left(S_{r}+S_{n}\right)$ and curvature, the contribution to flame stretch associated with $\left(S_{r}+S_{n}\right) \nabla \cdot \vec{N}$ becomes a non-linear function of curvature subject to a correlation between $\left(S_{r}+S_{n}\right)$ and curvature. The effect is not appreciable for the planar case but when the flame has an inherent mean curvature (in the present case, a mean positive curvature) the effect cannot be ignored. This indicates the need to account for the curvature dependence in $\left(S_{r}+S_{n}\right)$ for flame propagation modelling, even in the thin reaction zones regime. It should be noted that an increase in the turbulence intensity may lead to a decreasing correlation between $\left(S_{r}+S_{n}\right)$ and curvature, however under those circumstances the flame may be approaching the broken reaction zones regime. ${ }^{15}$ In the present study the turbulence intensity is not varied, and clearly this is an avenue for further investigation.

\section{CONCLUSIONS}

DNS has been carried out in three dimensions for turbulent flame kernels of different initial radius. A pair of back-to-back statistically planar flames was considered as a special case of a flame kernel with infinite radius. It has been observed that the mean positive curvature in the flame kernel cases significantly affects the mean behaviour of displacement speed $S_{d}$, mainly through the tangential diffusion component $S_{t}$. Based on the mean behaviour of $S_{d}$ it can be inferred that the assumption $\left\langle\rho S_{d}\right\rangle_{s}=\rho_{0} S_{L}$ is only valid when the flame is statistically planar. The pdfs of displacement speed show a 
significant increase in the probability of finding negative values of $S_{d}$ with decreasing kernel radius, which indicates that a minimum kernel size is required to sustain combustion. This is in accordance with the findings of previous ignition studies. ${ }^{16}$ The curvature dependence of $S_{d}$ and its various components $\left(S_{r}, S_{n}\right.$ and $\left.S_{t}\right)$ has been studied in terms of their joint pdfs with curvature. The local displacement speed is shown to be negatively correlated with curvature. However, the correlation is shown to be non-linear in nature as a result of non-linear curvature response of $\left(S_{r}+S_{n}\right)$. Furthermore, it was observed that the curvature response of $S_{r}$ and $S_{n}$ is essentially determined by the nature of the curvature dependence of the SDF $|\nabla c|$. For the kernel cases, $S_{r}$ and $S_{n}$ are correlated with curvature with increasing positive correlation strength for decreasing kernel radius, whereas in statistically planar flames both $S_{r}$ and $S_{n}$ are only weakly correlated with curvature. As a result of this correlation the Markstein diffusivity $D_{M}$ based on displacement speed is shown to decrease with decreasing kernel radius. The behaviour of $S_{r}$ and $S_{n}$ in the case of statistically planar flames is consistent with previous DNS studies. ${ }^{2,3,7,34}$ The strong curvature dependence of both $S_{r}$ and $S_{n}$ in the kernel cases indicates a need for modelling of the contribution of $\left(S_{r}+S_{n}\right)$ to displacement speed.

The above analysis does not address the influence of differential diffusion and detailed chemistry, nor does it deal with the effects of high frequency turbulent straining and flame quenching at very small kernel radius. Some of these issues will be addressed in detail in future work. 


\section{ACKNOWLEGEMENTS}

This work is supported by a fellowship within the Postdoc Program of the German Academic Exchange Service (DAAD). 


\section{REFERENCES}

${ }^{1}$ D.C.Haworth, Applications of turbulent combustion modelling, Turbulent Combustion, Lecture Series 2003-04, von Karman Institute for Fluid Dynamics, March 17-21,2003 (2003).

${ }^{2}$ T.Echekki and J.H.Chen, "Unsteady Strain rate and Curvature Effects in Turbulent Premixed Methane-Air Flames",Combust. Flame, 106,184 (1996).

${ }^{3}$ N.Peters, P.Terhoeven, J.H.Chen and T.Echekki, "Statistics of Flame Displacement Speeds from Computations of 2-D Unsteady Methane-Air Flames", Proc. of Combust. Instit., 27,833(1998).

${ }^{4}$ J.H.Chen and H.G.Im, "Correlation of Flame Speed with Stretch in Turbulent Premixed Methane/Air Flames", Proc. of Combust. Instit., 29,920 (1998).

${ }^{5}$ C.J.Rutland and A. Trouvé, "Direct Simulations of Premixed Turbulent Flames with Nonunity Lewis numbers",Combust. Flame, 94,41 (1993).

${ }^{6}$ R.S. Cant, C.J.Rutland, and A. Trouvé, "Statistics for laminar flamelet modelling", Proceedings of the Summer Program 1990, Center for Turbulence Research, Stanford, 271(1990).

${ }^{7}$ N.Chakraborty and S. Cant, "Unsteady Effects of Strain rate and Curvature on Turbulent Premixed Flames in Inlet-Outlet Configuration”, Combust.Flame 137,129 (2004).

${ }^{8}$ T. Echekki, T. Poisnot, T. Baritaud, M. Baum, "Modeling and Simulation of Turbulent Flame Kernel Evolution” in: T. Baritaud, T. Poinsot, M. Baum (Eds.) Direct Numerical Simulation for Turbulent Reacting Flows, Edition Technip Paris, 123 (1996).

${ }^{9}$ K.W. Jenkins, R.S. Cant, "Curvature effects on flame kernels in a turbulent environment”, Proc. Combust. Inst. ,29 2023 (2002). 
${ }^{10}$ D.Thévénin, O.Gicquel, J.D. Charentenay, R.Hilbert, and D.Veynante, “Two-Versus Three-Dimensional Direct Simulations of Turbulent Methane Flame Kernels Using Realisitc Chemistry" Proc. Combust. Inst., 29, 2031, (2002).

${ }^{11}$ D.Thévénin, "Three-Dimensional Direct Simulations and Structure of Expanding Turbulent Methane Flames" Proc. Combust. Inst. 30,629 (2005).

${ }^{12}$ R.J.M. Bastiaans, J.A. van Oijen, S.M. Martin, L.P.H. de Goey, H. Pitsch, "DNS of lean premixed turbulent spherical flames with a Flamelet Generated Manifold" Annual research Briefs 2004, Center for Turbulence Research, 257, (2004).

${ }^{13}$ J.A. Van Oijen, G.R.A. Groot, R. J.M. Bastiaans, L.P.H. de Goey, “A flamelet analysis of the burning velocity of premixed turbulent expanding flames", Proc. Combust. Inst. 30, $657(2005)$.

${ }^{14}$ S.B.Pope, "The evolution of surfaces in turbulence", Int. J. Engng. Sci., 26:5, 445(1988).

${ }^{15}$ N. Peters, Turbulent Combustion (Cambridge University press, Cambridge, UK, (2000).

${ }^{16}$ M. Baum, T. Poinsot, "Effects of Mean Flow on Premixed Flame Ignition", Combust. Sci. and Tech. 106, 19, (1995).

${ }^{17}$ C.F. Kaminski, J. Hult, M. Aldén, S. Lindenmaier, A. Dreizler, U. Maas, M. Baum, "Spark Ignition of Turbulent Methane/Air Mixtures Revealed by Time Resolved Planar Laser-Induced Fluorescence and Direct Numerical Simulations“, Proc. Combust. Inst. 28, $399,(2000)$. 
${ }^{18}$ S. Gashi, J. Hult, K.W. Jenkins, N. Chakraborty, S. Cant, C.F. Kaminski, “Curvature and Wrinkling of Premixed Flame Kernels-Comparisons of OH PLIF and DNS data", Proc. Combust. Inst., 30, 809 (2005).

${ }^{19}$ W.Kollmann, and J.H.Chen, "Pocket formation and the flame surface density equation", Proc. of Combust. Instit., 27,927(1998).

${ }^{20}$ K.W. Jenkins and R.S Cant, "DNS of turbulent flame kernels" in Proc. Second AFOSR Conf. on DNS and LES, (Kluwer Academic Publishers, 1999) pp. 192-202.

${ }^{21}$ A.A Wray, "Minimal storage time advancement schemes for spectral methods", unpublished report, NASA Ames Research Center, California, (1990).

${ }^{22}$ R.S.Rogallo, "Numerical experiments in homogeneous turbulence”, NASA Technical Memorandum 81315, NASA Ames Research Center, California (1981).

${ }^{23}$ T. Poinsot, S.K. Lele, "Boundary conditions for direct simulation of compressible viscous flows", J. Comp. Phys., 101, 104 (1992).

${ }^{24}$ M.Boger, D.Veynante, H.Boughanem, and A.Trouvé, "Direct Numerical Simulation analysis of flame surface density concept for Large Eddy Simulation of turbulent premixed combustion”, Proc. of Combust. Instit., 27,917(1998).

${ }^{25}$ A.Trouvé and T.J.Poinsot, "The evolution equation for flame surface density in turbulent premixed combustion", J.Fluid Mech., 244,405(1992).

${ }^{26}$ J.F. Driscoll, and A.Gulati, "Measurement of various terms in turbulent kinetic balance within a flame and comparison with theory", Combust. Flame 72,131(1988).

${ }^{27}$ R.K.Cheng, I.G.Shepherd, and L.Talbot, "Reaction rates in Premixed Turbulent Flames and their relevance to Turbulent Burning Speed", Proc. of Combust. Instit., 22, 771 (1988). 
28 T.C.Chew, K.N.C.Bray, and R.E. Britter, "Spatially resolved flamelet statistics for reaction rate modelling", Combust. Flame 80,65 (1990).

${ }^{29}$ N. Chakraborty and R.S. Cant, "Effects of Strain Rate and Curvature on Surface Density Function transport in turbulent premixed flames in the Thin Reaction Zones Regime" Phys. Fluids, 17, 65108-1 (2005).

${ }^{30}$ I.R.Gran, T.Echekki and J.H.Chen, ,"Negative flame speed in an unsteady 2-D premixed flame: A computational study", Proc. of Combustion Institute, 26, 211 (1996).

${ }^{31}$ B.Renou, A.Boukhalfa, D.Peuchberty and M. Trinité, "Effects of stretch on the local structure of freely propagating premixed low-turbulent flames with various Lewis numbers", Proc. of Combust. Instit., 27,841(1998).

${ }^{32}$ D.C.Haworth and T.J.Poinsot, "Numerical simulations of Lewis number effects in turbulent premixed flames", J.Fluid Mech., 244,405(1992).

${ }^{33}$ W.T.Ashurst, A.R. Kerstein, R.M. Kerr, and C. H. Gibson, “ Alignment of vorticity and scalar gradient with strain rate in Navier-Stokes turbulence", Physics of Fluids A, 30, 2343 (1987).

${ }^{34}$ T.Echekki and J.H.Chen, "Analysis of the Contribution of Curvature to Premixed Flame Propagation", Combust. Flame, 118, 303 (1996). 


\section{TABLES}

\begin{tabular}{|c|c|}
\hline Case (Grid size) & $r_{o} / \delta_{t h}$ \\
\hline$A\left(230^{3}\right)$ & $\infty$ \\
\hline B $\left(230^{3}\right)$ & 2.91 \\
\hline $\mathrm{C}\left(230^{3}\right)$ & 2.49 \\
\hline D $\left(230^{3}\right)$ & 2.10 \\
\hline$E\left(230^{3}\right)$ & 1.74 \\
\hline $\mathrm{F}\left(230^{3}\right)$ & 1.42 \\
\hline Cases A-F & $\begin{array}{c}\operatorname{Pr}=0.7 ; L e=1.0 ; \beta=6.0 ; \tau=3.0 ; M a=S_{L} / a_{0}=0.014159 ; \\
\quad \gamma=C_{p} / C_{v}=1.4 ; u^{\prime} / S_{L}=7.5 ; L_{11} / \delta_{L}=1.7 ; \mathrm{Re}_{t}=32\end{array}$ \\
\hline
\end{tabular}

Table I: Parameters of the DNS database. The initial kernel radius $r_{0}$ is defined as the radius of the region in which $c=1.0$.

\begin{tabular}{|c|c|}
\hline Case & $D_{M} / D$ \\
\hline $\mathbf{A}$ & 1.00 \\
\hline $\mathbf{B}$ & 0.67 \\
\hline $\mathbf{C}$ & 0.62 \\
\hline $\mathbf{D}$ & 0.64 \\
\hline $\mathbf{E}$ & 0.54 \\
\hline $\mathbf{F}$ & 0.46 \\
\hline
\end{tabular}

Table II: Markstein diffusivity ( $\left.D_{M}\right)$ normalized by the mean value of diffusivity (D) on the $c=0.8$ isosurface. 


\begin{tabular}{|c|c|c|c|c|c|}
\hline $\mathbf{1}$ & $\mathbf{2}$ & $\mathbf{3}$ & $\mathbf{4}$ & $\mathbf{5}$ & $\mathbf{6}$ \\
\hline Case & $\begin{array}{c}\text { Corr. } \\
|\nabla c|-a_{T}\end{array}$ & $\begin{array}{c}\text { Corr. } \\
|\nabla c|-\kappa_{m}\end{array}$ & $\begin{array}{c}\text { Corr. } \\
S_{r}-\kappa_{m}\end{array}$ & $\begin{array}{c}\text { Corr. } \\
S_{n}-\kappa_{m}\end{array}$ & $\begin{array}{c}\text { Corr. } \\
\left(S_{r}+S_{n}\right)-\kappa_{m}\end{array}$ \\
\hline $\mathbf{A}$ & 0.47 & -0.15 & 0.10 & -0.21 & 0.095 \\
\hline $\mathbf{B}$ & 0.72 & -0.66 & 0.66 & 0.02 & 0.45 \\
\hline $\mathbf{C}$ & 0.68 & -0.69 & 0.63 & 0.15 & 0.52 \\
\hline $\mathbf{D}$ & 0.82 & -0.72 & 0.77 & 0.29 & 0.62 \\
\hline $\mathbf{E}$ & 0.81 & -0.80 & 0.83 & 0.46 & 0.70 \\
\hline $\mathbf{F}$ & 0.64 & -0.80 & 0.79 & 0.45 & 0.76 \\
\hline
\end{tabular}

Table III: Correlation coefficients obtained for all DNS cases from the joint pdfs of: (column 2) $|\nabla c|$ and $a_{T}$; (column 3) $|\nabla c|$ and $\kappa_{m}$; (column 4) $S_{r}$ and $\kappa_{m}$; (column 5) $S_{r}$ and (column 6) $\left(S_{r}+S_{n}\right)$ and $\kappa_{m}$. 


\section{FIGURE CAPTIONS}

Figure 1: Contours of reaction progress variable in the $x-z$ plane at $y=0.5$. (a) Case A $\left(r_{o} / \delta_{t h}=\propto\right)$; (b) Case C $\left(r_{o} / \delta_{t h}=2.49\right)$; (c) Case F $\left(r_{o} / \delta_{t h}=1.42\right)$.

Figure 2: Variation of reaction rate and molecular diffusion rate terms in the reaction progress variable transport equation (see eqs. 2 and 5). Reaction rate $=\dot{w}$, molecular diffusion rate $=\nabla \cdot(\rho D \nabla c)$, normal diffusion term $=\vec{N} \cdot \nabla(\rho D \vec{N} \cdot \nabla c)$, tangential diffusion term $=-(\rho D \nabla \cdot \vec{N}|\nabla c|)$. All the terms are ensemble averaged over $c$ isosurfaces. (a) Case $\mathrm{A}\left(r_{o} / \delta_{t h}=\propto\right)$; (b) Case C $\left(r_{o} / \delta_{t h}=2.49\right)$; (c) Case F $\left(r_{o} / \delta_{t h}=1.42\right)$.

Figure 3: Variation of combined reaction rate and molecular diffusion rate $(\dot{w}+\nabla \cdot(\rho D \nabla c))$; combined reaction rate and normal component of molecular diffusion rate $(\dot{w}+\vec{N} . \nabla(\rho D \vec{N} . \nabla c)) ; \rho_{0} S_{L}|\nabla c|$ plotted across the flame brush. All the terms are ensemble averaged over $c$ isosurfaces. (a) Case A $\left(r_{o} / \delta_{t h}=\propto\right)$; (b) Case $\mathrm{C}$ $\left(r_{o} / \delta_{t h}=2.49\right) ;(\mathrm{c})$ Case F $\left(r_{o} / \delta_{t h}=1.42\right)$.

Figure 4: The variation of (density weighted) mean values of displacement speed components $S_{d} / S_{L},\left(S_{r}+S_{n}\right) / S_{L}, S_{t} / S_{L}, \rho\left(S_{r}+S_{n}\right) / \rho_{0} S_{L}$ and $\rho S_{d} / \rho_{0} S_{L}$ across the flame brush. (a) Case A $\left(r_{o} / \delta_{t h}=\propto\right)$; (b) Case C $\left(r_{o} / \delta_{t h}=2.49\right)$; (c) Case F $\left(r_{o} / \delta_{t h}=1.42\right)$

Figure 5: Pdfs of normalised displacement speed $\left(S_{d} / S_{L}\right)$ on isosurfaces of progress variable. The value of progress variable runs from $c=0.1$ to 0.9 in steps of 0.2 . (a) Case $\mathrm{A}\left(r_{o} / \delta_{t h}=\propto\right)$; (b) Case C $\left(r_{o} / \delta_{t h}=2.49\right)$; (c) Case F $\left(r_{o} / \delta_{t h}=1.42\right)$. 
Figure 6: Pdfs of normalised components of displacement speed on isosurfaces of progress variable for cases $\mathrm{A}, \mathrm{C}$ and F. (a) Pdf of reaction component $S_{r}$; (b) Pdf of normal diffusion component $S_{n}$; (c) Pdf of tangential diffusion component $S_{t}$. Displacement speed components are normalised with respect to unstrained laminar flame speed $S_{L}$.

Figure 7: Joint pdf of $S_{d}$ and mean curvature on the isosurface at $c=0.8$. (a) Case A $\left(r_{o} / \delta_{t h}=\propto\right)$; (b) Case $\mathrm{C}\left(r_{o} / \delta_{t h}=2.49\right)$; (c) Case F $\left(r_{o} / \delta_{t h}=1.42\right)$. Note that $S_{d}$ is normalised using the unstrained laminar flame speed $S_{L}$ and mean curvature is normalised with respect to reference length scale.

Figure 8: Joint pdf of SDF and mean curvature on the isosurface at $c=0.8$. (a) Case A $\left(r_{o} / \delta_{t h}=\propto\right)$; (b) Case C $\left(r_{o} / \delta_{t h}=2.49\right)$; (c) Case F $\left(r_{o} / \delta_{t h}=1.42\right)$. Both SDF and mean curvature are normalised with respect to reference length scale.

Figure 9: Joint pdf of tangential strain rate $\left(a_{T}\right)$ and mean curvature on the isosurface at $c$ $=0.8$. (a) Case A $\left(r_{o} / \delta_{t h}=\propto\right)$; (b) Case C $\left(r_{o} / \delta_{t h}=2.49\right)$; (c) Case F $\left(r_{o} / \delta_{t h}=1.42\right)$. Note that both quantities are normalised with respect to reference velocity and length scales.

Figure 10: Joint pdf of dilatation $(\nabla \cdot \vec{u})$ and mean curvature on the isosurface at $c=0.8$. (a) Case A $\left(r_{o} / \delta_{t h}=\propto\right)$; (b) Case $\mathrm{C}\left(r_{o} / \delta_{t h}=2.49\right)$; (c) Case $\mathrm{F}\left(r_{o} / \delta_{t h}=1.42\right)$. The quantities are normalised with respect to reference velocity and length scales.

Figure 11: Pdfs of normal strain rate $\left(a_{n}=N_{i} N_{j} \partial u_{i} / \partial x_{j}\right)$ on isosurfaces of progress variable. The value of progress variable runs from $c=0.1$ to 0.9 in steps of 0.2. (a) Case A $\left(r_{o} / \delta_{t h}=\propto\right)$; (b) Case C $\left(r_{o} / \delta_{t h}=2.49\right)$; (c) Case F $\left(r_{o} / \delta_{t h}=1.42\right)$. Normal strain 
rate is normalised with respect to appropriate combination of reference velocity and length scales.

Figure 12: Joint pdf of the reaction component of displacement speed $S_{r}$ and mean curvature on the isosurface at $c=0.8$. (a) Case $\mathrm{A}\left(r_{o} / \delta_{t h}=\propto\right)$; (b) Case $\mathrm{C}$ $\left(r_{o} / \delta_{t h}=2.49\right) ;(\mathrm{c})$ Case $\mathrm{F}\left(r_{o} / \delta_{t h}=1.42\right)$.

Figure 13: Joint pdf of the normal diffusion component of displacement speed $S_{n}$ and mean curvature on the isosurface at $c=0.8$. (a) Case $\mathrm{A}\left(r_{o} / \delta_{t h}=\propto\right)$; (b) Case $\mathrm{C}$ $\left(r_{o} / \delta_{t h}=2.49\right) ;(\mathrm{c})$ Case F $\left(r_{o} / \delta_{t h}=1.42\right)$.

Figure 14: Joint pdf of the combined reaction and normal diffusion component of displacement speed $S_{r}+S_{n}$ and mean curvature on the isosurface at $c=0.8$. (a) Case A $\left(r_{o} / \delta_{t h}=\propto\right)$; (b) Case C $\left(r_{o} / \delta_{t h}=2.49\right)$; (c) Case F $\left(r_{o} / \delta_{t h}=1.42\right)$. 
Figure 1

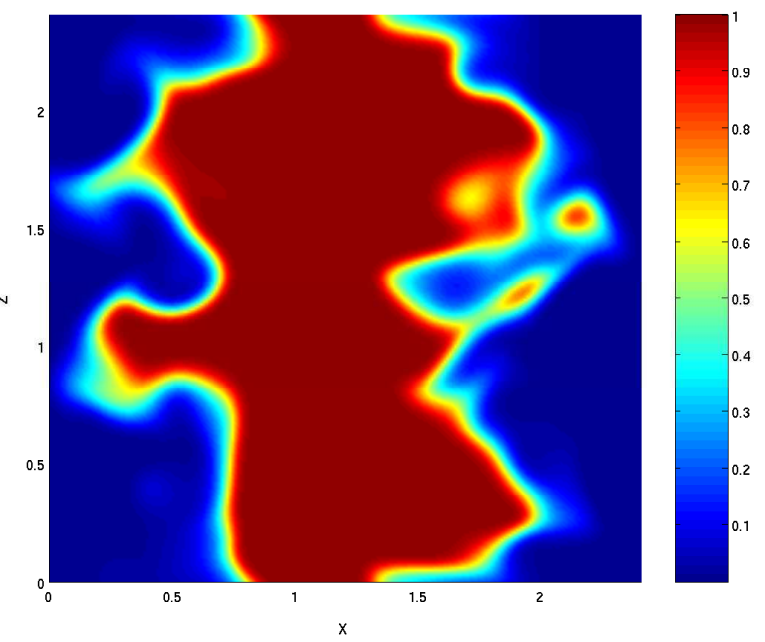

a

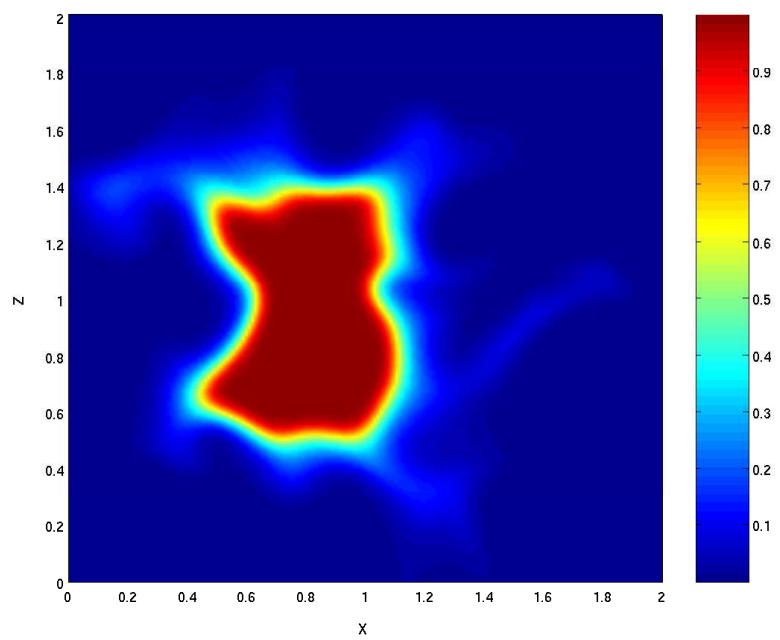

b

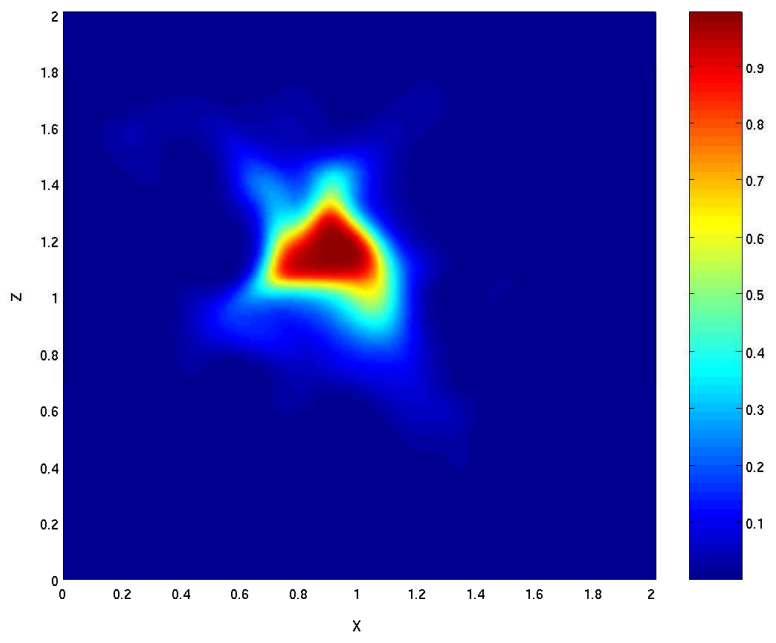


Figure 2

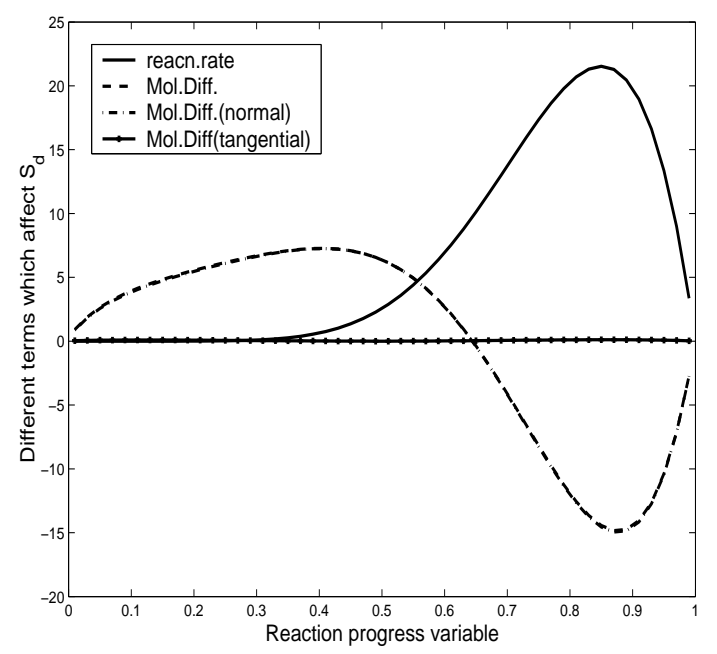

a

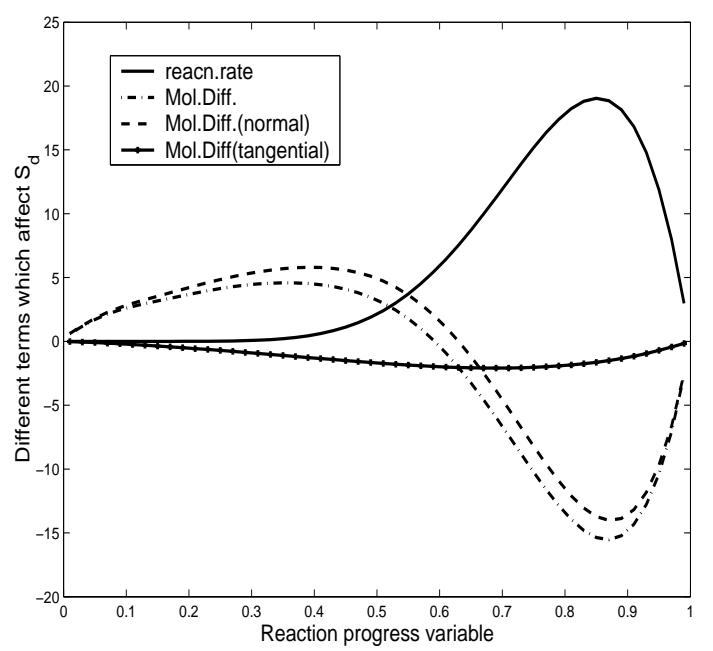

b

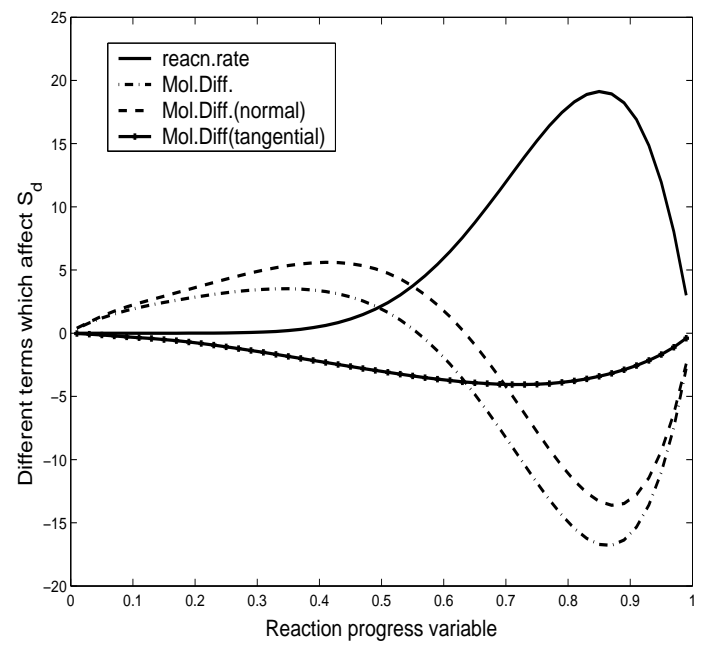




\section{Figure 3}

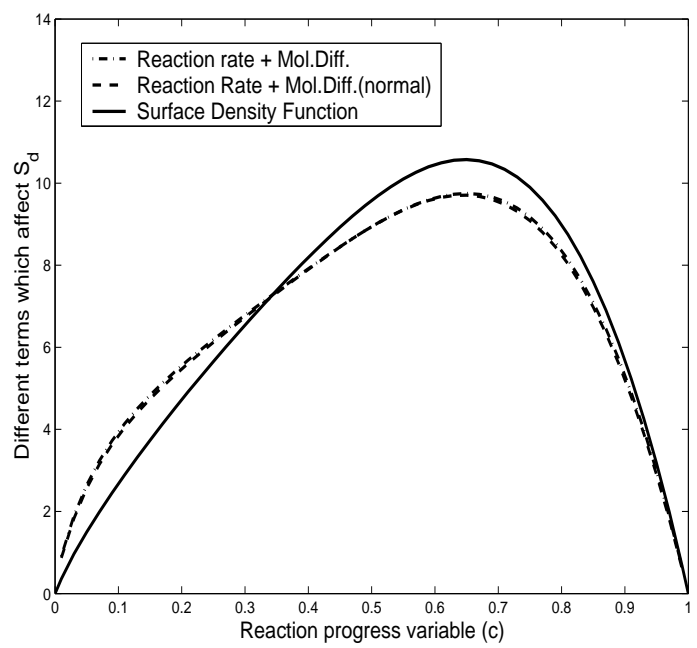

a

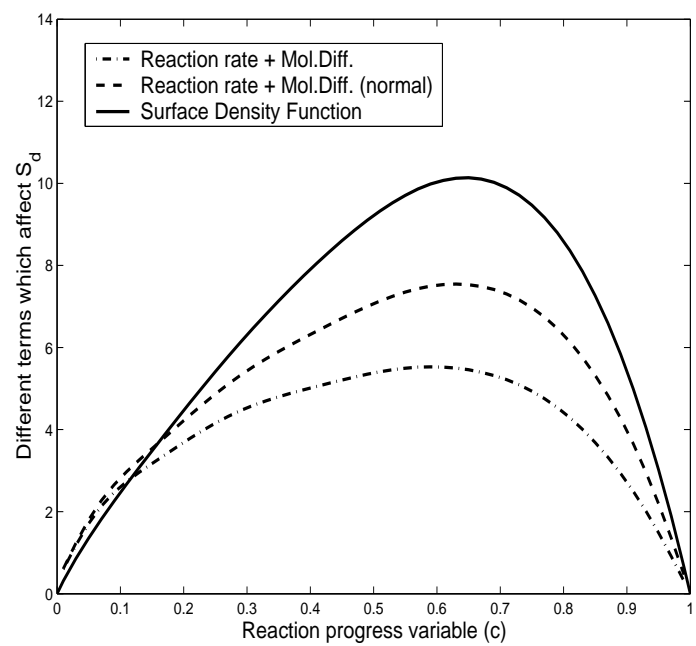

b

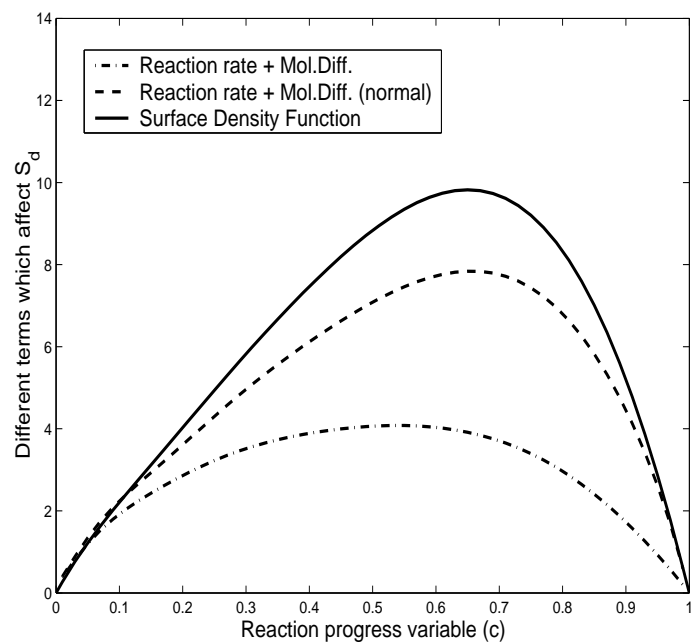




\section{Figure 4}

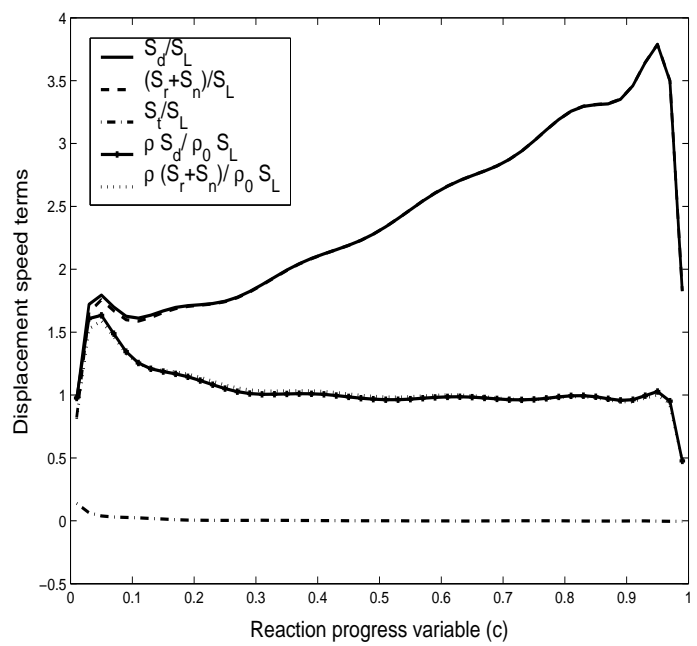

a

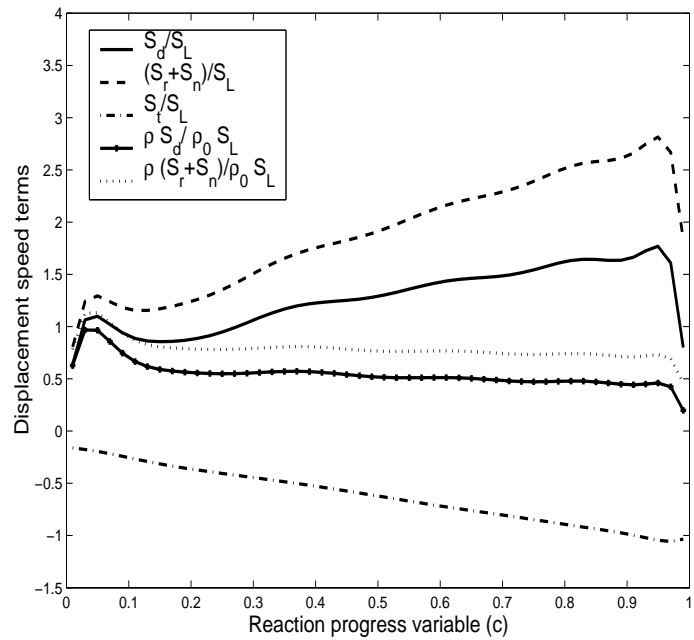

b

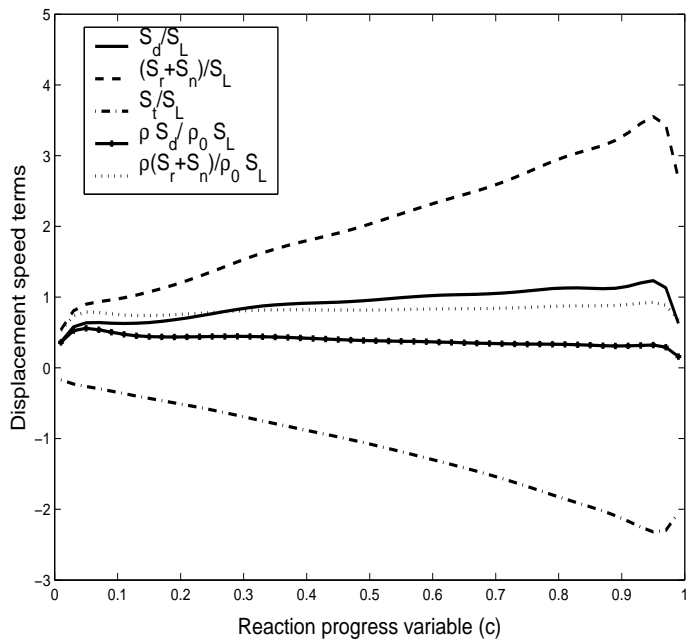


Figure 5

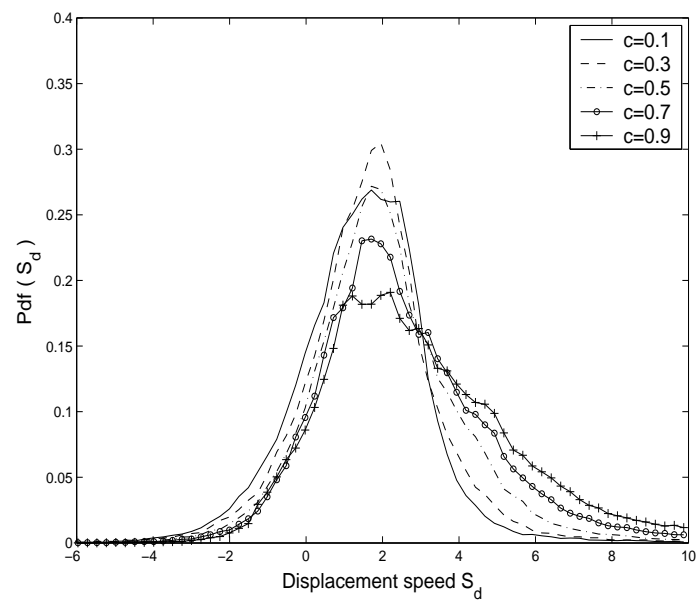

a

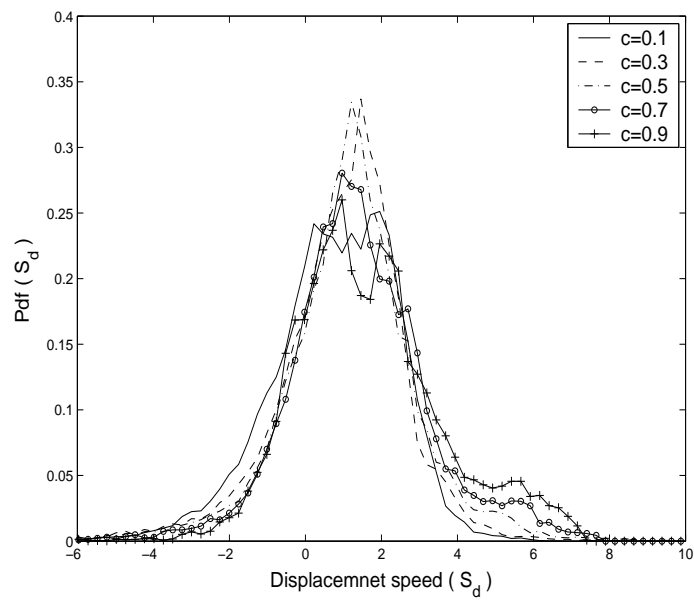

b

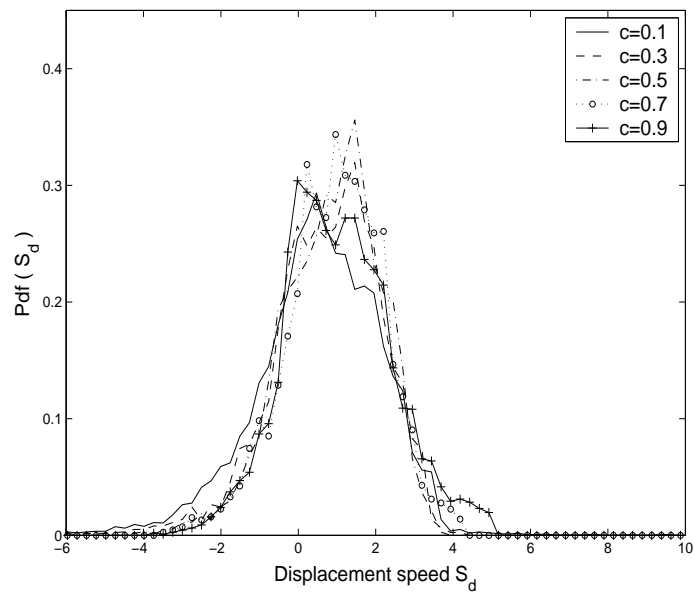

c 


\section{Figure 6}

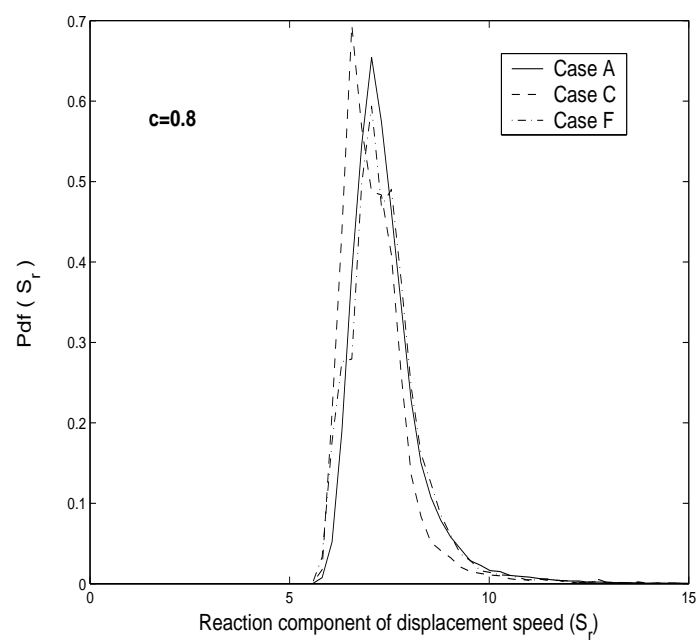

a

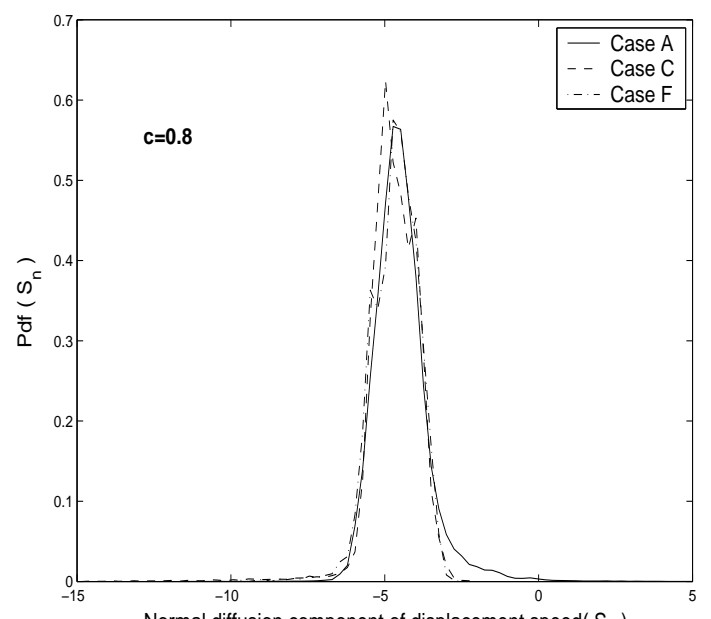

Normal diffusion component of displacement speed $\left(S_{n}\right)$

b

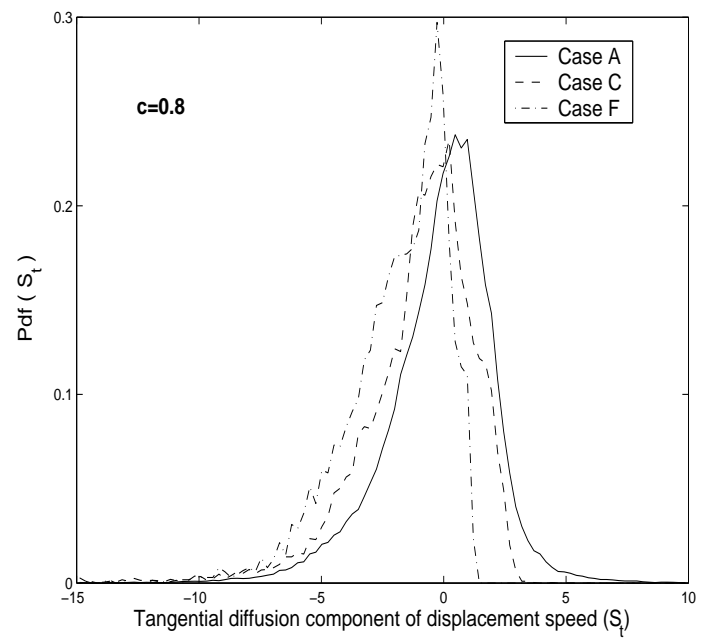


Figure 7

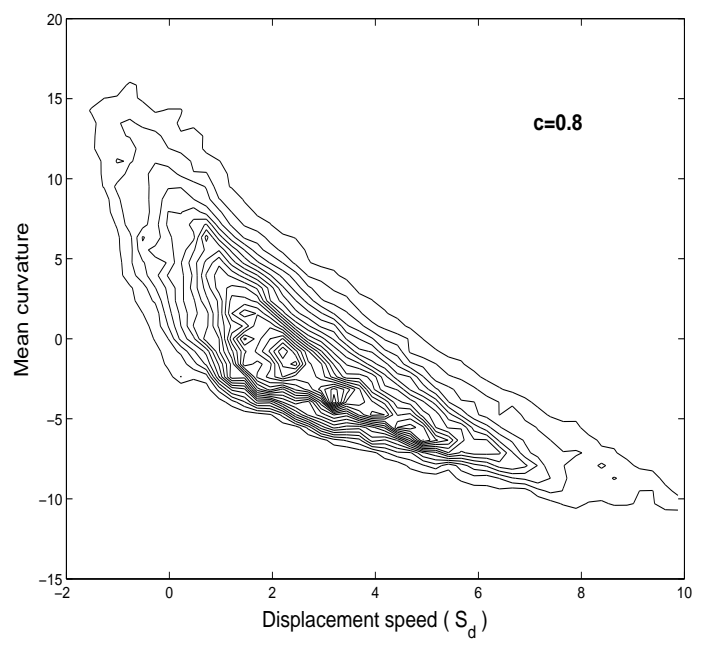

a

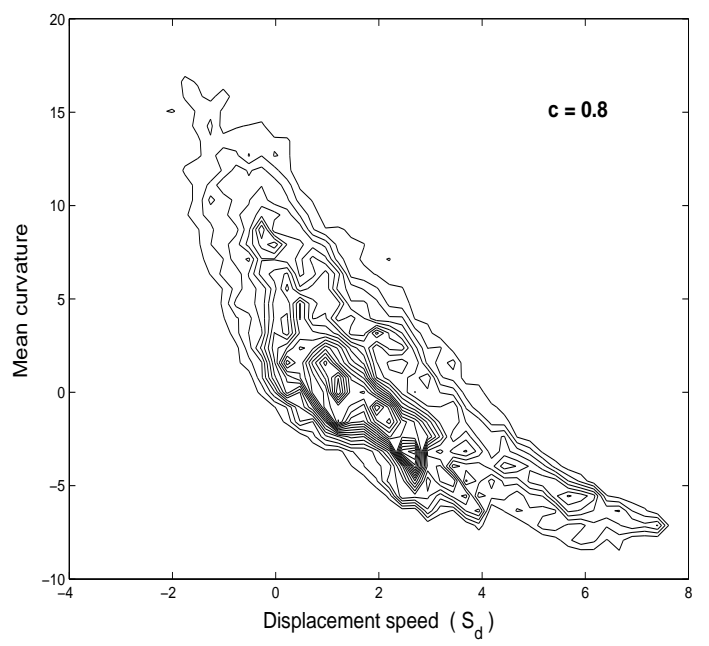

b

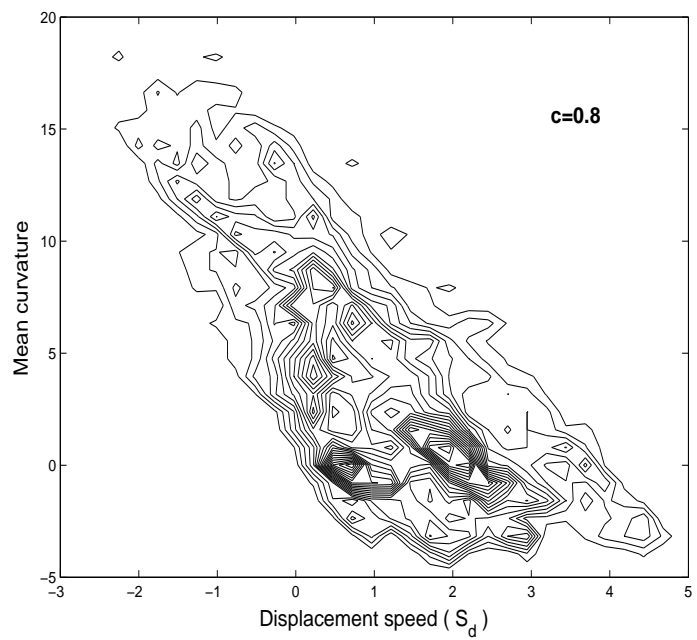

c 
Figure 8

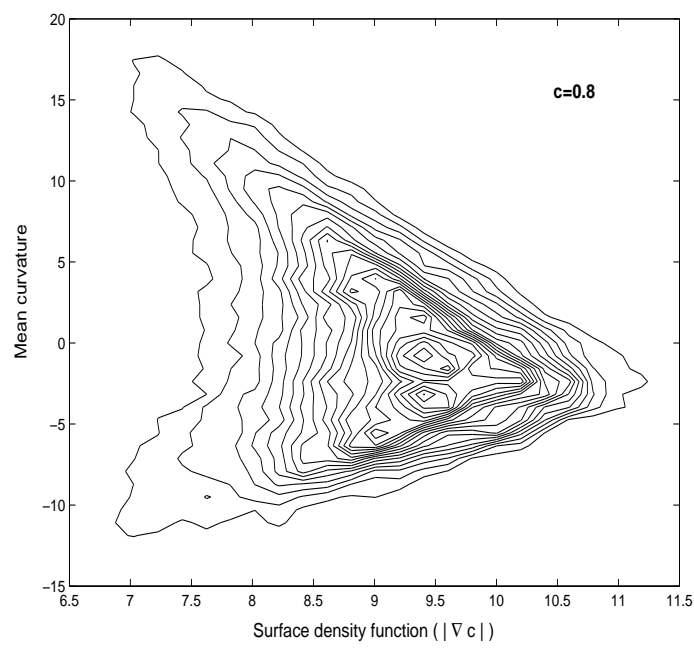

a

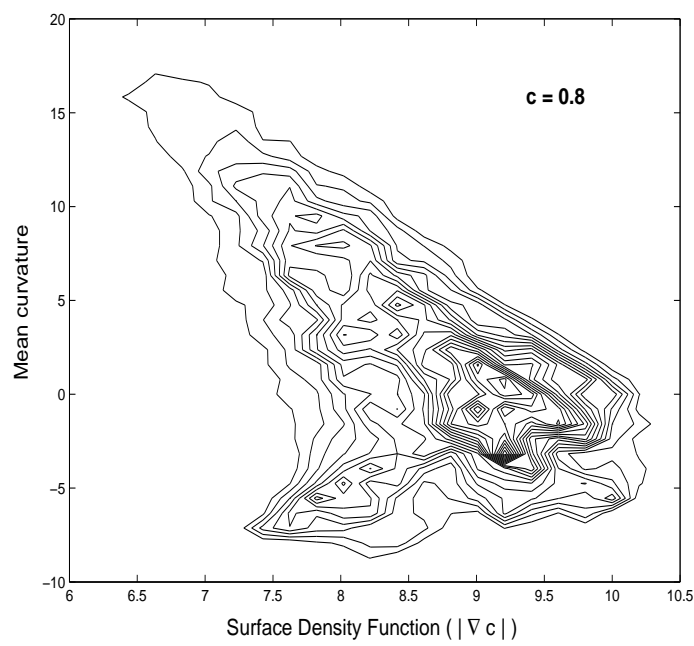

b

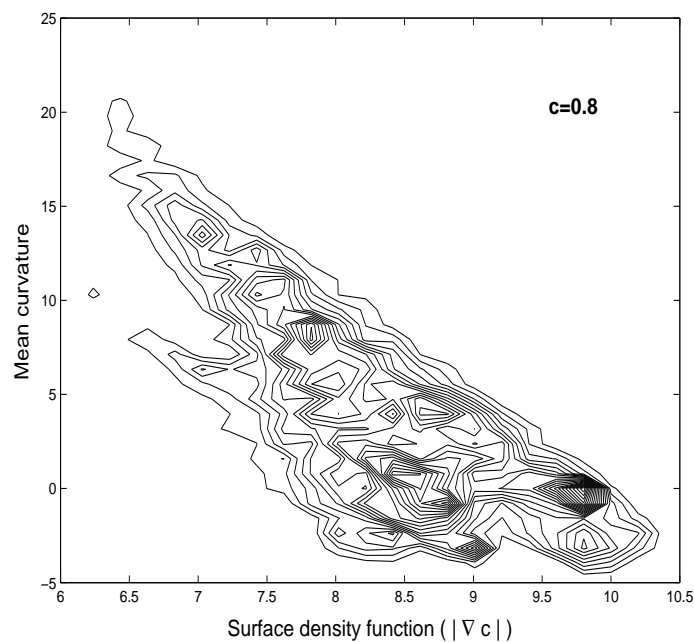

c 
Figure 9

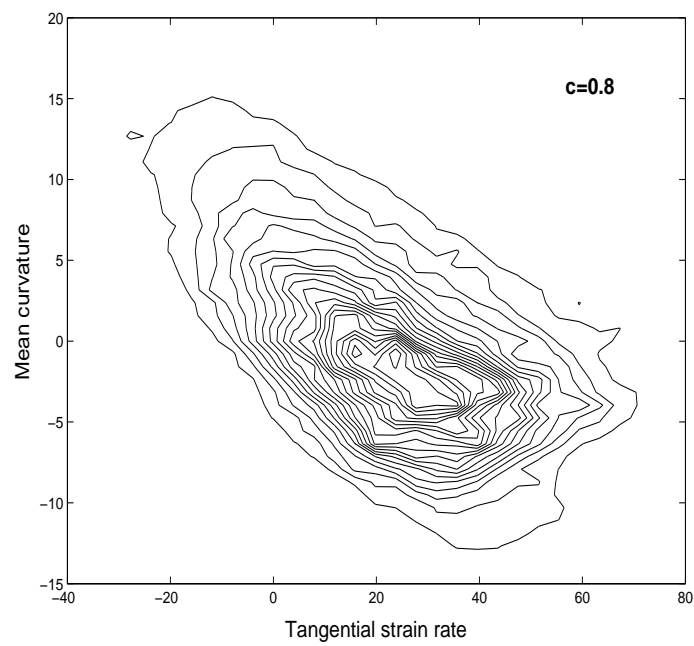

a

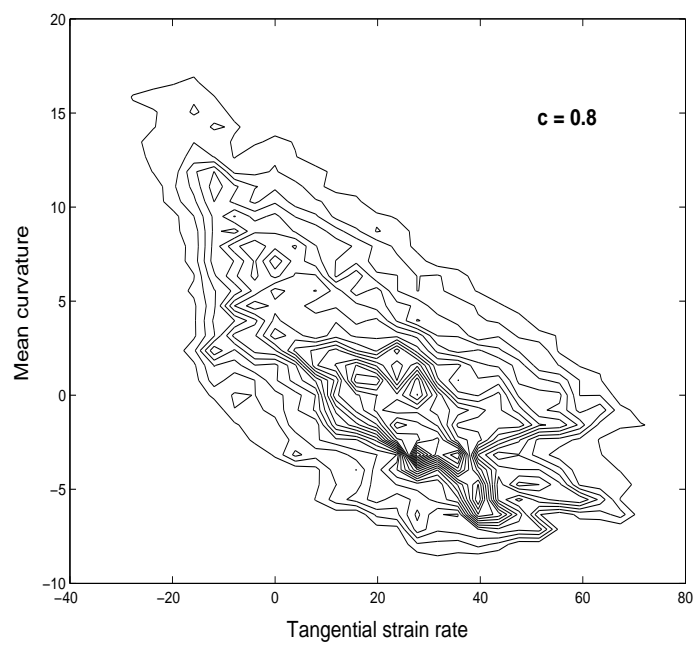

b

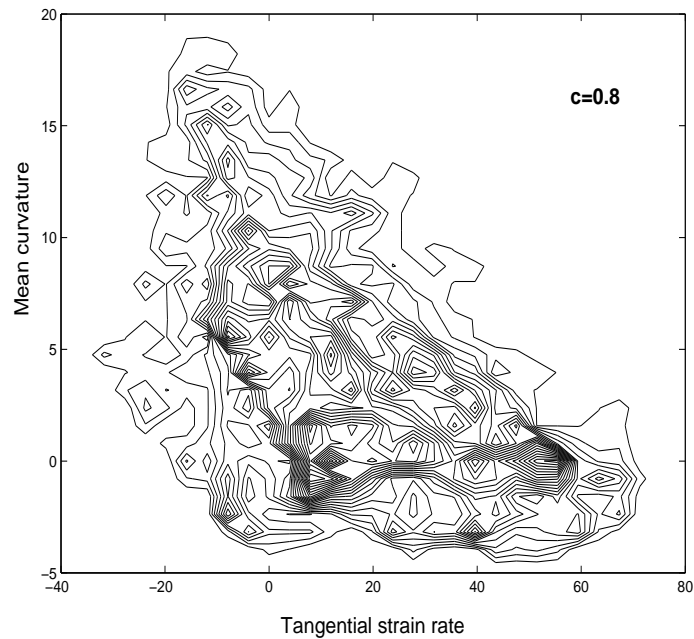

c 
Figure 10

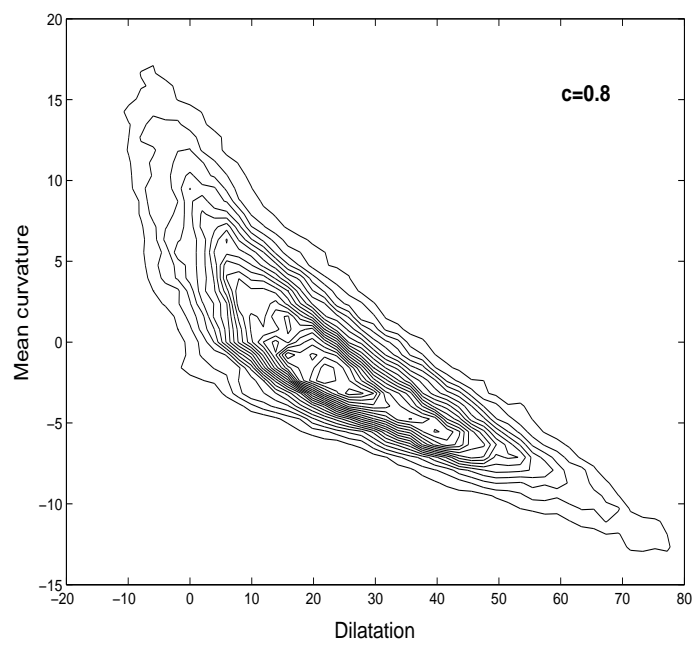

a

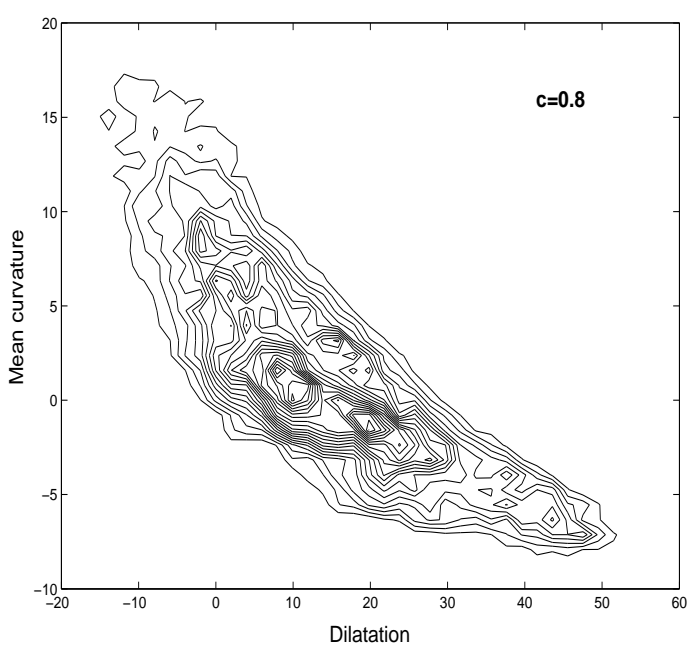

b

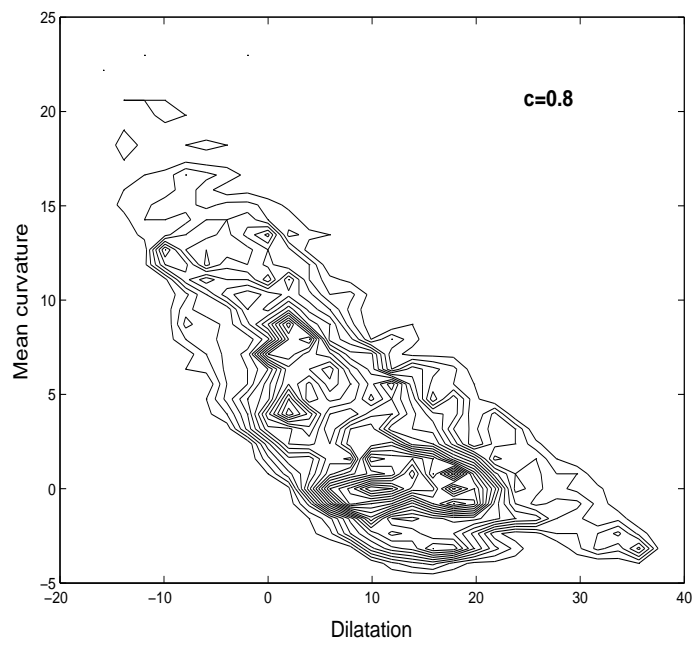

c 
Figure 11

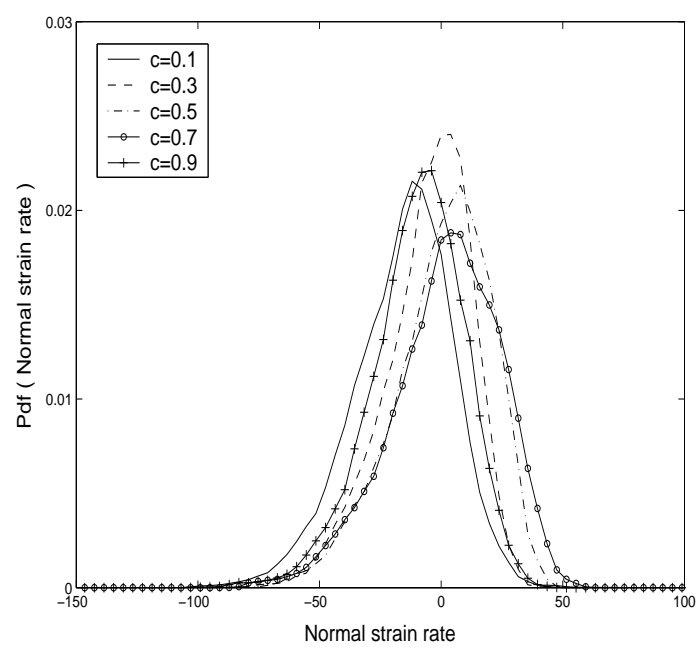

a

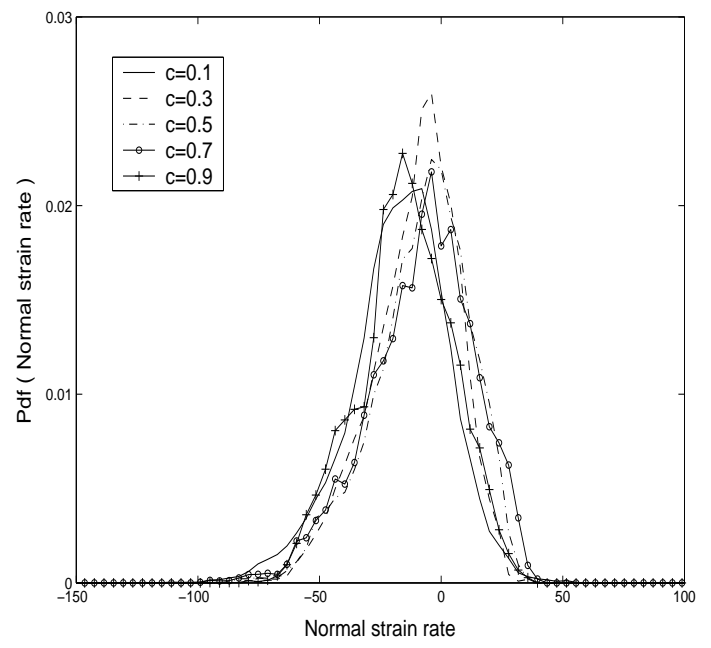

b

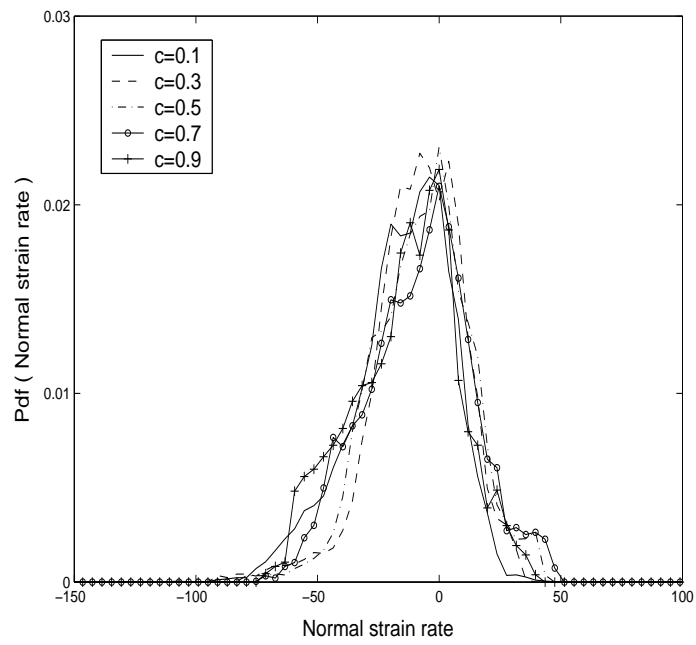

c 
Figure 12

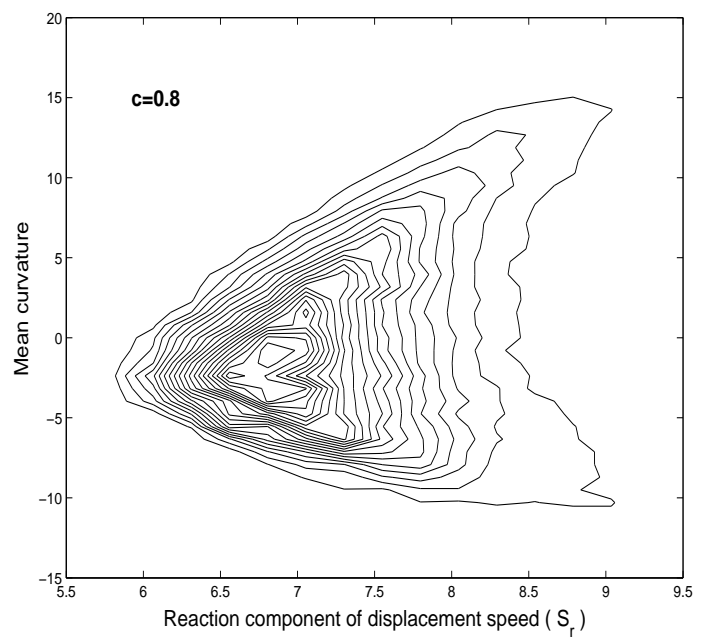

a

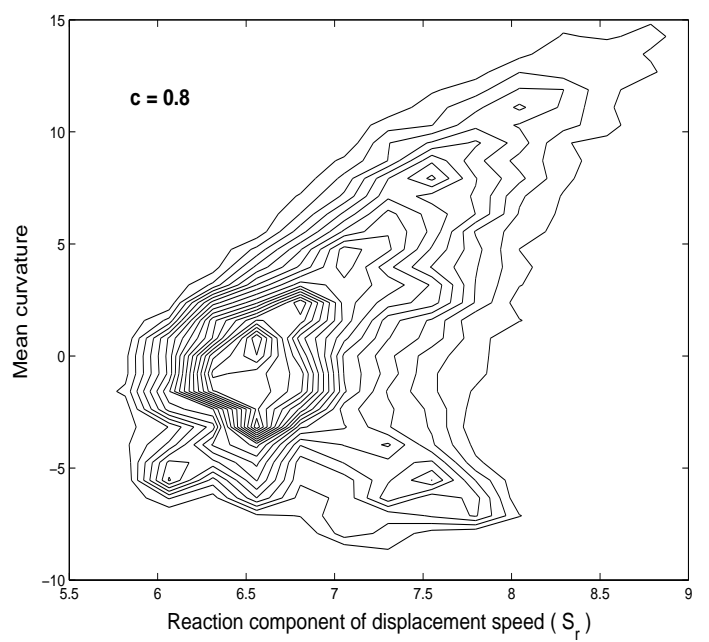

b

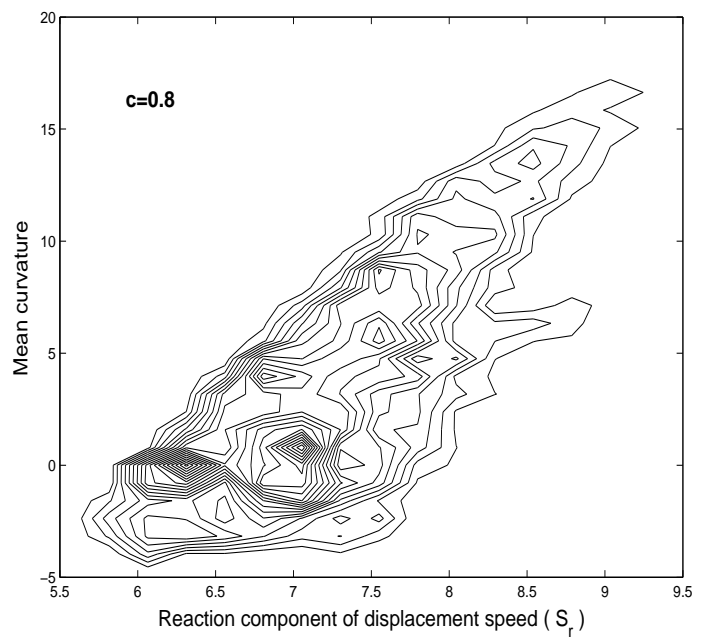


Figure 13

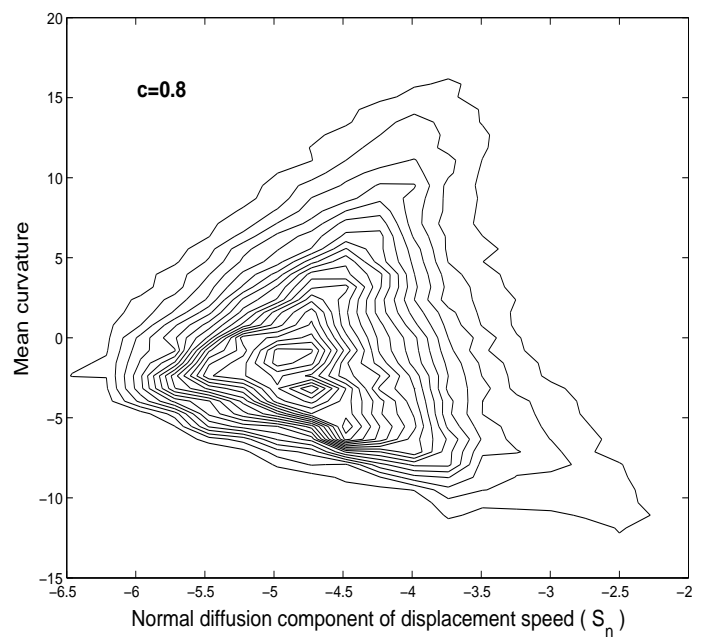

a

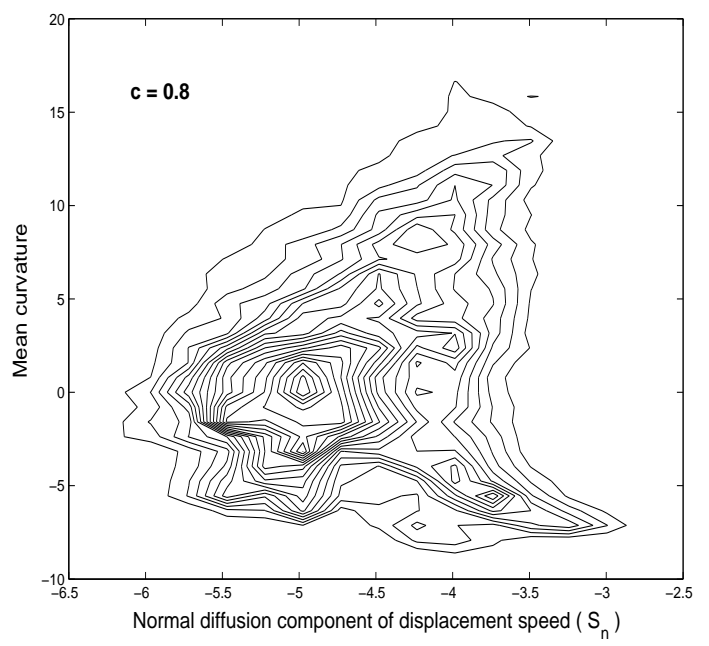

b

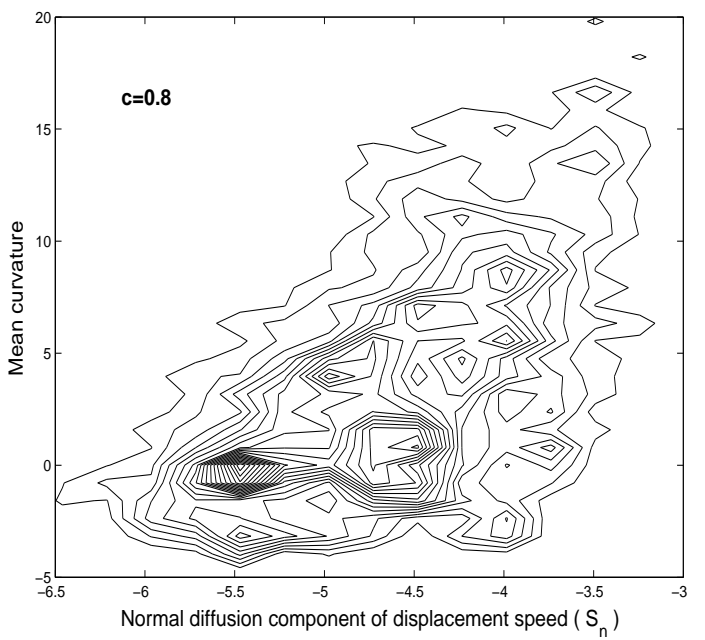


Figure 14

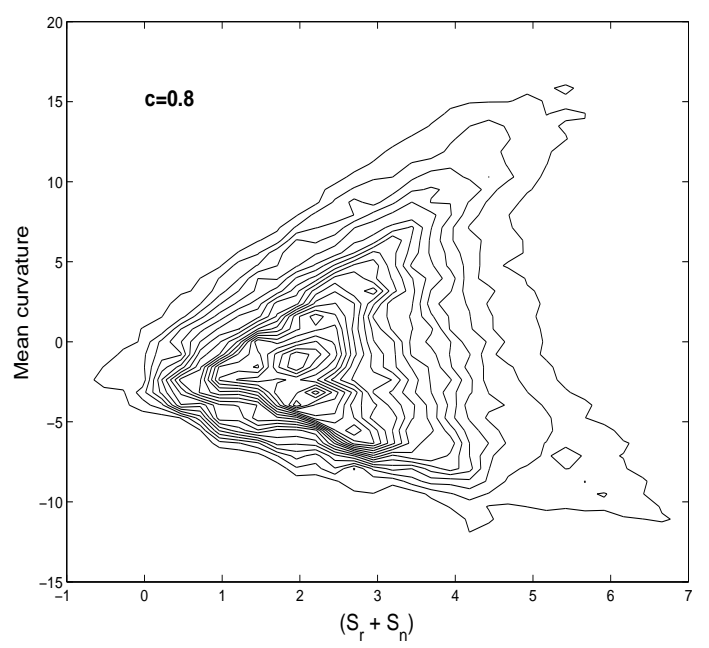

a

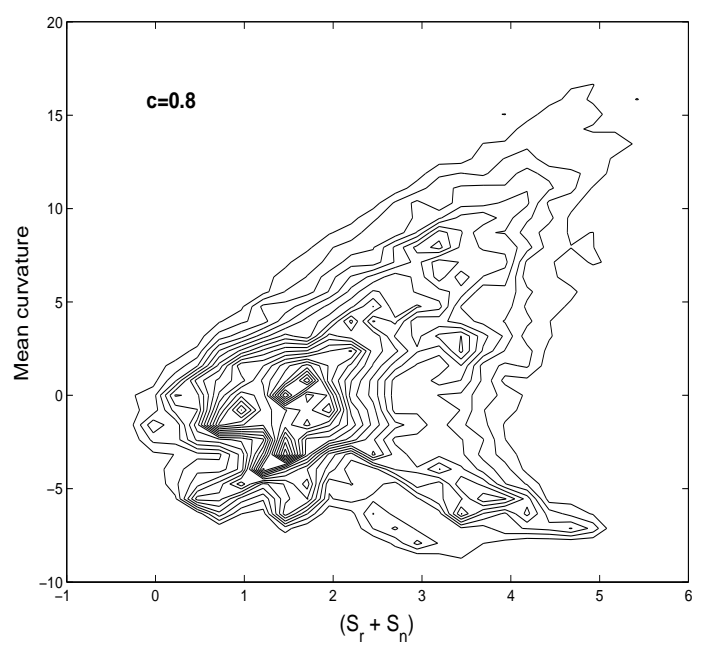

b

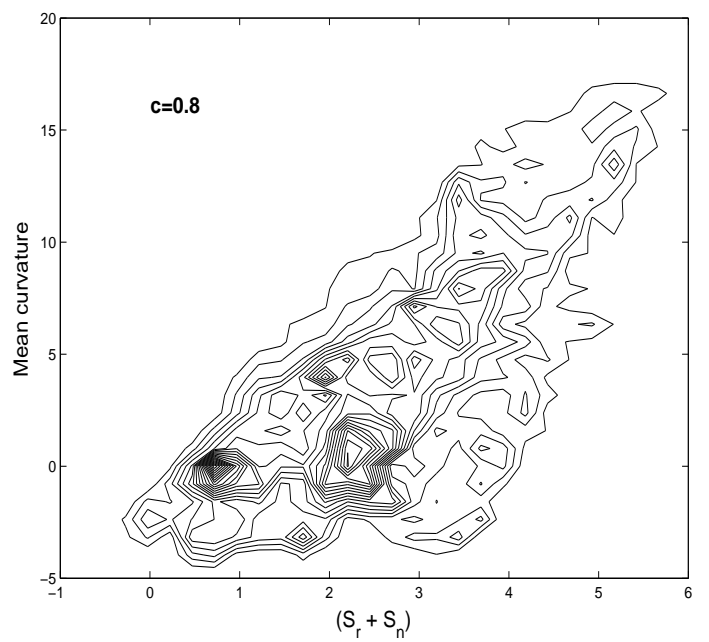

c 


\section{Effects of initial radius on the}

propagation of premixed flame kernals

in a turbulent environment

Klein, M.

AIP

Klein M, Chakraborty N, Jenkins K, Cant R. Effects of initial radius on the propagation of premixed flame kernals in a turbulent environment. Physics of Fluids, Volume 18, 2006, Article number 055102, pp. 102-115

https://doi.org/10.1063/1.2196092

Downloaded from Cranfield Library Services E-Repository 